\title{
FGFR2 signaling underlies p63 oncogenic function in squamous cell carcinoma
}

\author{
Matthew R. Ramsey, ${ }^{1}$ Catherine Wilson, ${ }^{1}$ Benjamin Ory, ${ }^{1}$ S. Michael Rothenberg, ${ }^{2}$ \\ William Faquin, ${ }^{2}$ Alea A. Mills, ${ }^{3}$ and Leif W. Ellisen ${ }^{1}$
}

\begin{abstract}
${ }^{1}$ Massachusetts General Hospital Cancer Center and Harvard Medical School, Boston, Massachusetts, USA.
2Department of Pathology, Massachusetts General Hospital and Harvard Medical School, Boston, Massachusetts, USA.

${ }^{3}$ Cold Spring Harbor Laboratory, Cold Spring Harbor, New York, USA.
\end{abstract}

\begin{abstract}
Oncogenic transcription factors drive many human cancers, yet identifying and therapeutically targeting the resulting deregulated pathways has proven difficult. Squamous cell carcinoma (SCC) is a common and lethal human cancer, and relatively little progress has been made in improving outcomes for SCC due to a poor understanding of its underlying molecular pathogenesis. While SCCs typically lack somatic oncogene-activating mutations, they exhibit frequent overexpression of the p53-related transcription factor $\mathrm{p} 63$. We developed an in vivo murine tumor model to investigate the function and key transcriptional programs of p63 in SCC. Here, we show that established SCCs are exquisitely dependent on p63, as acute genetic ablation of p63 in advanced, invasive SCC induced rapid and dramatic apoptosis and tumor regression. In vivo genome-wide gene expression analysis identified a tumor-survival program involving p63-regulated FGFR2 signaling that was activated by ligand emanating from abundant tumor-associated stroma. Correspondingly, we demonstrate the therapeutic efficacy of extinguishing this signaling axis in endogenous SCCs using the clinical FGFR2 inhibitor AZD4547. Collectively, these results reveal an unanticipated role for $\mathrm{p} 63$-driven paracrine FGFR2 signaling as an addicting pathway in human cancer and suggest a new approach for the treatment of SCC.
\end{abstract}

\section{Introduction}

Squamous cell carcinoma (SCC) is a treatment-refractory malignancy arising within the epithelium of the skin, lung, esophagus, and upper aerodigestive tract (so-called head and neck SCC, HNSCC). SCC is largely a carcinogen-induced tumor, as major risk factors include tobacco, alcohol, and sun exposure. Little progress has been made in improving overall survival from SCC during the past 30 years, in part because of a limited understanding of the molecular pathobiology of this disease (1). SCCs do not commonly harbor somatic oncogene-activating mutations, but instead are associated with frequent mutational inactivation of tumor suppressor pathways including $p 53$ and $\mathrm{NOTCH}$, the therapeutic implications of which remain to be established $(2,3)$. In addition, although the EGFR is an attractive therapeutic target that is overexpressed or amplified in a subset of cases, only limited success with EGFR-directed therapy has been achieved in SCC despite intensive efforts over many years $(4,5)$. Recent systematic sequencing studies of HNSCC have confirmed the remarkable rarity of tumor-associated somatic mutations in well-established oncogenic drivers, which include HRAS (4\% of cases) and the PI3K catalytic subunit PIK3CA (7\% of cases) $(2,3)$. Thus, new approaches are needed to identify the key biological drivers in SCC in order to develop more effective and selective therapies for this disease.

One underexplored area for potential therapeutic intervention in SCC and other cancers involves deregulation of various lineagespecific transcription factors (6). The p53-related transcription factor $p 63$ (TP63), a master regulator of embryonic epithelial development, is subject to overexpression and/or genomic amplification in up to $80 \%$ of primary HNSCC tumors as well as in SCCs of the skin, lung, and esophagus $(7,8)$. Numerous p63-regulated

Conflict of interest: The authors have declared that no conflict of interest exists. Citation for this article: J Clin Invest. 2013;123(8):3525-3538. doi:10.1172/JCI68899. transcriptional target genes and programs have been identified through developmental and cell-culture studies, including differentiation, adhesion signaling, and senescence suppression (9-11). While several of these genes and pathways could in theory contribute to tumorigenesis, it is unknown which if any are relevant in the context of p63-expressing tumors in vivo. Indeed, the precise role of $\mathrm{p} 63$ in cancer may be context dependent, as loss rather than overexpression of $\mathrm{p} 63$ is associated with disease progression in some tumors, including bladder cancer (12). These seemingly paradoxical results could be in part attributable to distinct functions of different $\mathrm{p} 63$ protein variants. The $p 63$ locus is expressed as multiple isoforms, most notably through 2 promoters that produce $\mathrm{N}$-terminal variants either containing or lacking the $\mathrm{p} 53$-like transactivation domain (TAp63 or $\Delta \mathrm{Np} 63$, respectively) (13). Data from mouse models indeed support isoform-specific functions of p63 in both development and cancer. While germline inactivation of $p 63$ (14) or $\Delta N p 63$ (15) results in mice lacking skin and limbs and is perinatal lethal, selective TAp63 deficiency produces viable animals that exhibit an increase in metastatic tumors (16). Notably, the major p63 isoform expressed in stratified squamous epithelium and SCC is $\Delta \mathrm{Np} 63 \alpha(17,18)$.

The interactions between tumor cells and their microenvironment likely play an essential role in SCC pathogenesis, providing another fertile area for investigation and potential therapeutic intervention. Substantial data point to essential and possibly distinct roles for immune cells and stromal cells in SCC initiation and progression $(19,20)$. Of particular interest in recent years has been the contribution of tumor-associated fibroblasts. In many cancers, these cells have been shown to contribute to tumor proliferation, differentiation, and invasion as well as creation of a permissive environment for tumor formation and maintenance (20). Encasement of tumor cells within a dense fibrous stroma is a hallmark of HNSCC and other squamous tumors, and high 


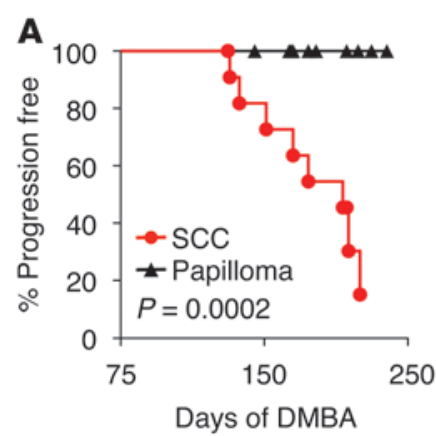

B

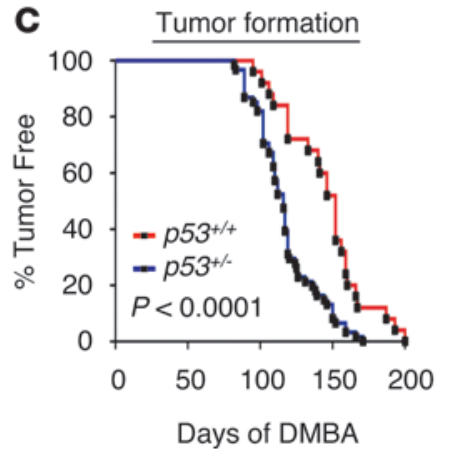

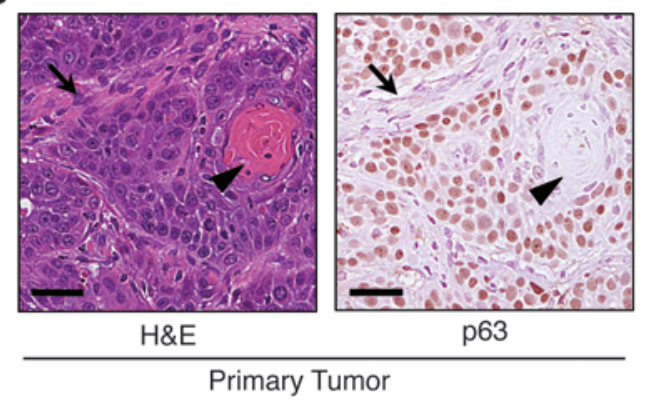

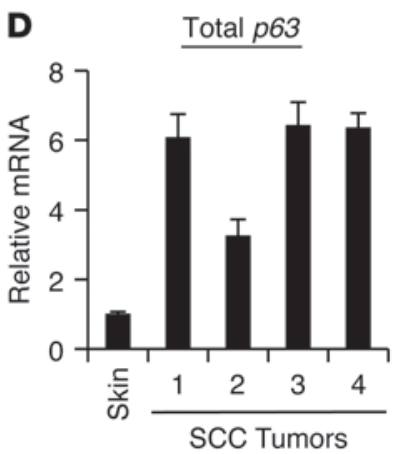

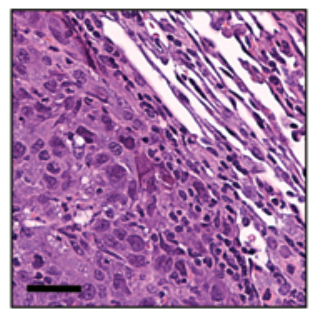

$H \& E$

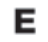

E

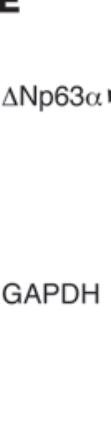

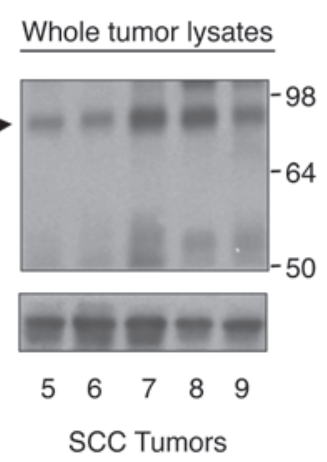

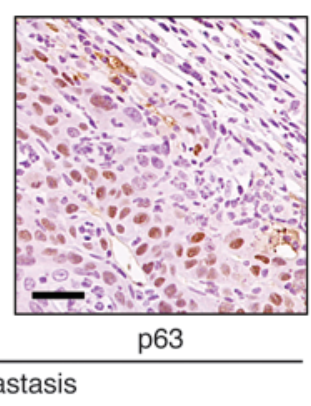

$\mathbf{F}$

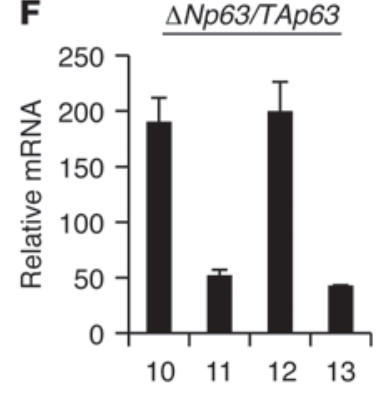

Figure 1

Murine SCC tumors share molecular features of human SCC. (A) Kaplan-Meier curve of progression of histologically defined papillomas compared with SCC tumors. Tumors were considered progressive when they passed $100 \mathrm{~mm}^{3}$ size threshold. (B) Primary and metastatic SCC tumors from DMBA-treated mice share the histologic features (H\&E) — note stromal elements (arrows) and differentiated keratin "pearls" (arrowheads) and nuclear p63 expression (IHC) of human SCC. Scale bars: $25 \mu \mathrm{m}$. (C) Haploinsufficiency for p53 reduces tumor latency following weekly DMBA treatment. $p 53^{+/+}, n=25, p 53^{+/-}, n=61$. $P$ value assessed by log-rank test. (D) Increased expression of $p 63$ mRNA in DMBA-induced SCCs versus normal skin. mRNA levels were assessed by QRT-PCR and normalized to $\beta$-actin. Error bars represent SEM. (E) Predominant expression of $\Delta$ Np63 $\alpha$ protein (arrowhead) in murine SCC tumors. Whole-tumor lysates were assessed for p63 expression using an antibody that detects all isoforms. GAPDH serves as a loading control. (F) $\triangle N p 63$ is overexpressed relative to TAp63 in murine SCC tumors. Whole tumors were disaggregated and sorted for CD49fhi ( $\alpha-6$ integrin) epithelial cells, and mRNA levels were assessed as in $\mathbf{D}$.

levels of stromal infiltrate are associated with a poor prognosis in these malignancies $(21,22)$. Despite the clinical and biological importance of tumor stroma in SCC, however, previous work has provided few therapeutically actionable insights and the precise mechanisms of this stromal contribution are poorly understood.

Given the potentially complex role of p63 in SCC, understanding its precise contribution to cancer pathogenesis in the autochthonous tumor context could provide major new insights into this disease. We therefore established an endogenous SCC model to test the effect of deleting all $p 63$ isoforms within established, invasive tumors. This experiment demonstrated exquisite dependence of SCC on high levels of p63. Extensive in vivo analysis revealed a deregulated paracrine FGFR2 signaling program that is controlled directly by $\mathrm{p} 63$, activated by stroma-derived ligand, and required for tumor survival. We then validated these findings and their therapeutic relevance using a clinical FGFR2 inhibitor to induce apoptosis of advanced endogenous tumors.

\section{Results}

Development and validation of a robust murine SCC model. SCC is a cancer caused by prolonged, repeated carcinogen exposure. Therefore, to generate a murine SCC model that would recapitulate the features of the human disease we employed a well-established protocol of weekly carcinogen (7,12-dimethyl-benz[a]anthracene [DMBA]) treatment in the oral cavity (23) or on the back (24-26).
These SCCs were readily distinguishable from nonmalignant papillomas in both appearance and size (Figure 1, A and B). Tumors were locally invasive and exhibited metastases to lymph nodes and lung in a subset of cases ( 13\%), (Figure 1B). These tumors shared the histologic features of human SCC, including variable degrees of ongoing squamous epithelial differentiation and high levels of nuclear p63 expression (Figure 1B). Similar to human SCC, these tumors also expressed robust levels of EGFR and cyclin D1 proteins (Supplemental Figure 1A; supplemental material available online with this article; doi:10.1172/JCI68899DS1), and resemble the recently described "classical SCC" that develops in carcinogentreated mice (27). Human SCCs frequently harbor inactivating mutations in p53 $(2,3,28)$, and p53 inactivation is important for SCC progression in genetic models of oral SCC, cooperating potently with events such as AKT activation (29). Consistent with these data, we found that SCC tumors arising in DMBAtreated $p 53^{+/-}$mice appeared at reduced latency compared with those occurring in WT mice (Figure 1C) $(30,31)$. These tumors showed evidence of p53 loss or mutation as do human SCC primary tumors and cell lines (Supplemental Figure 1). Like human SCCs, our murine tumors express higher levels of p63 mRNA and protein than normal skin, corresponding to the $\Delta \mathrm{Np} 63 \alpha$ isoform (Figure 1, D and E, and refs. 10, 18, 32). Using FACS of disaggregated tumors to isolate cells expressing the epithelialspecific $\alpha 6$-integrin (CD49f) (33), we observed levels of $\Delta N p 63$ 
A

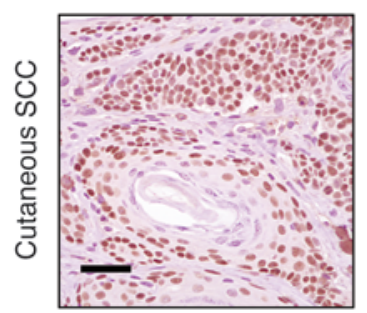

Keratin 14

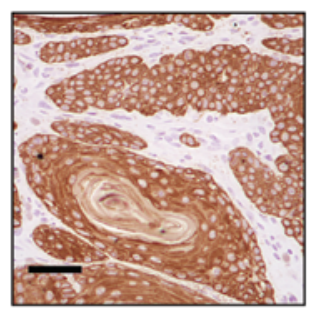

B

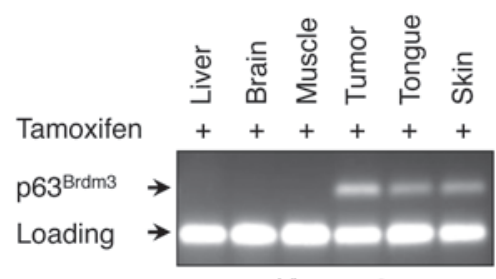

p63 $3^{L /}$ K14-CreER
C $\underline{\mathrm{p} 63 \mathrm{IHC}}$
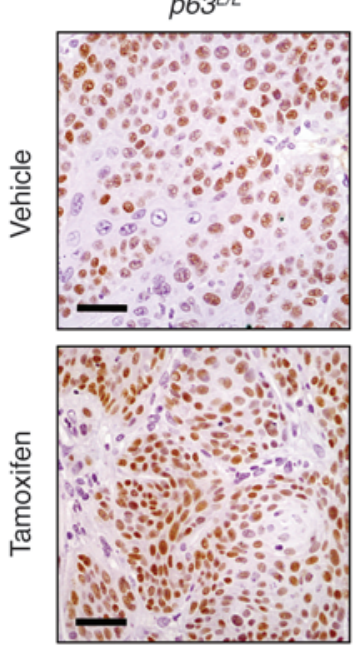

p63 $3^{L /}$ K14-CreER
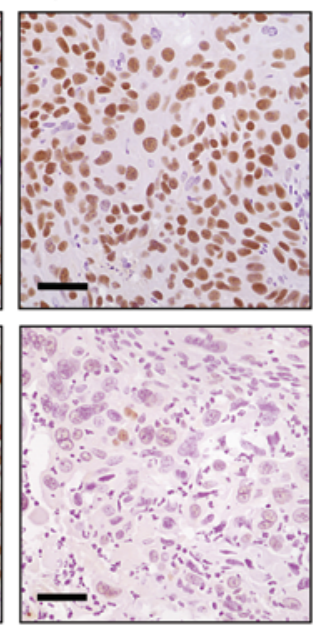

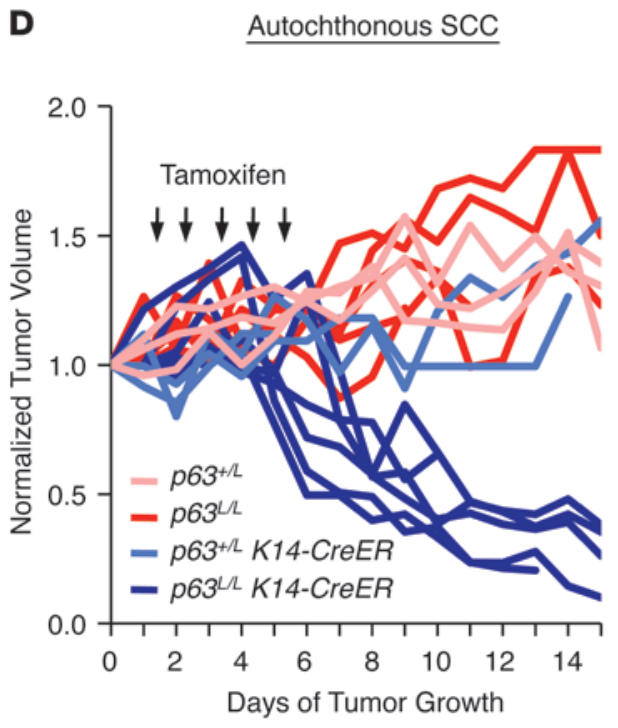

E

$p 63^{L / 2}$

p63 Kᄂ K14-CreER
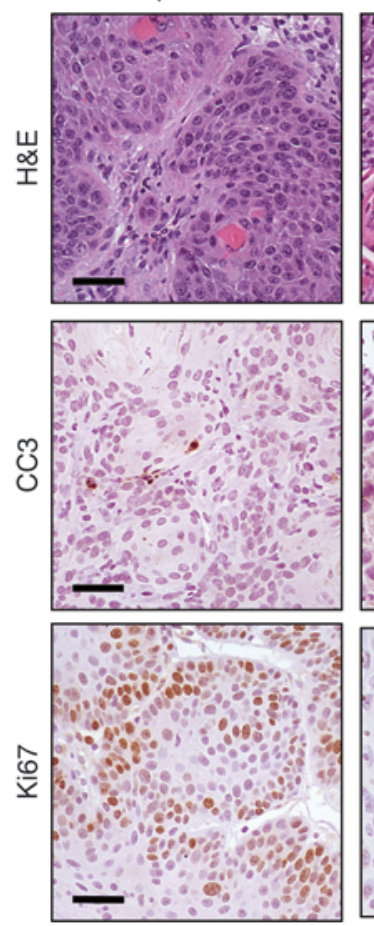
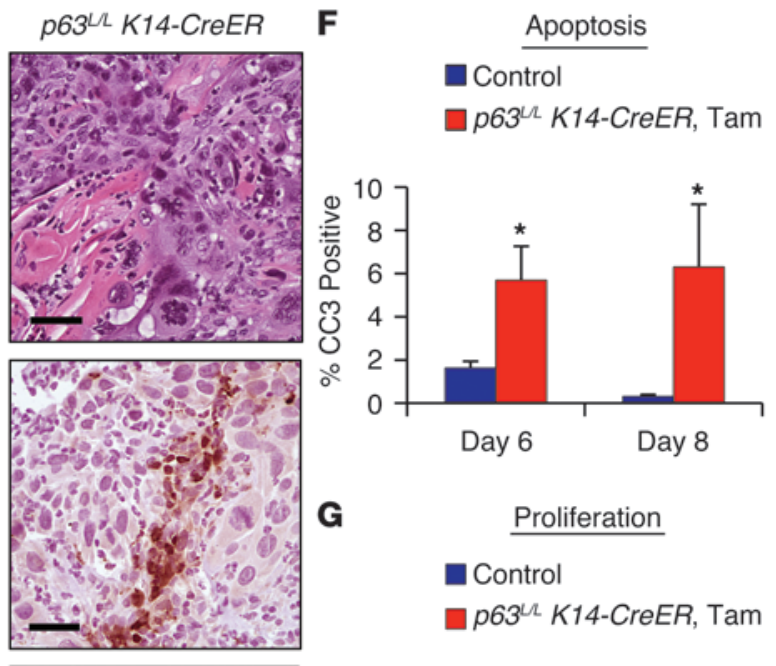

G

Proliferation

Control

p63几 K14-CreER, Tam

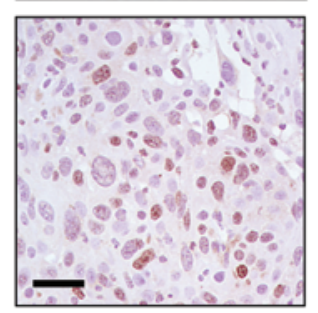

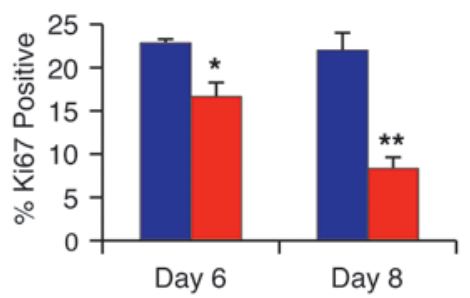

\section{Figure 2}

p63 is required for maintenance of autochthonous SCC tumors in vivo. (A) IHC of K14 and p63 in SCC tumors. Scale bars: $25 \mu \mathrm{m}$. (B) PCR analysis of DNA from tissues of a p63L/L K14-CreER mouse treated with daily i.p. injection of $100 \mathrm{mg} / \mathrm{kg}$ tamoxifen for 5 consecutive days. The p63 ${ }^{\mathrm{Brdm} 3}$ allele generated after excision and primer locations are shown in Supplemental Figure 2A. (C) IHC of p63 protein expression in tumors of indicated genotypes 72 hours after treatment with vehicle or tamoxifen as above. Scale bars: $25 \mu \mathrm{m}$. (D) Volume of cutaneous tumors in $p 63^{+/ L}$ $(n=4)$, p63 $3^{L / L}(n=6), p 63^{+/ L} K 14-C r e E R(n=3)$, or $p 63^{L / L} K 14-C r e E R(n=6)$ mice following tamoxifen treatment (arrows). Tumor volume was normalized to size 1 day prior to treatment. $P<0.0001$ for $p 63^{L / L} K 14-C r e E R$ compared with each control group as assessed by multiple measures ANOVA. (E) Representative histology (H\&E) and IHC staining for Ki67 and cleaved caspase 3 (CC3) of tumors from tamoxifen-treated p63L/L or p63L/L K14-CreER mice. Scale bar: $25 \mu \mathrm{m}$. (F) Quantification of cleaved caspase 3 staining in control versus tamoxifen-treated $p 63^{L / L}$ K14-CreER tumors, shown as percentage of field staining positive. ${ }^{*} P<0.05$ as determined by Mann-Whitney test. (G) Quantification of Ki67-positive cells in control versus tamoxifen-treated $K 14-C r e E R$ tumors. ${ }^{*} P<0.05$; ${ }^{*} P<0.01$ as determined by Student's unpaired $t$ test. Error bars represent SEM. See Methods for details of controls in $\mathbf{F}$ and $\mathbf{G}$. 
A

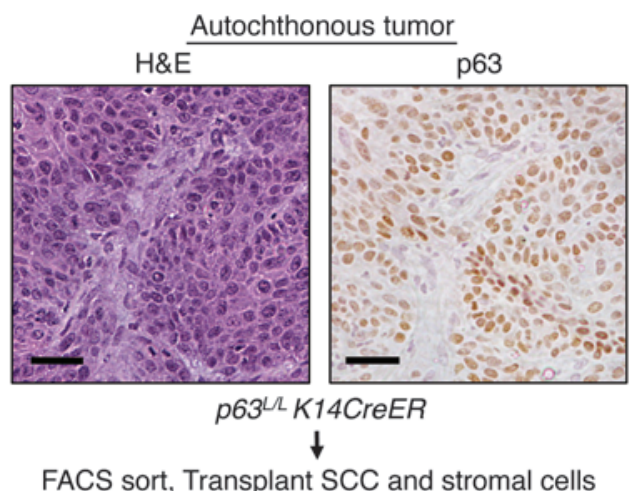

FACS sort, Transplant SCC and stromal cells

$\downarrow$

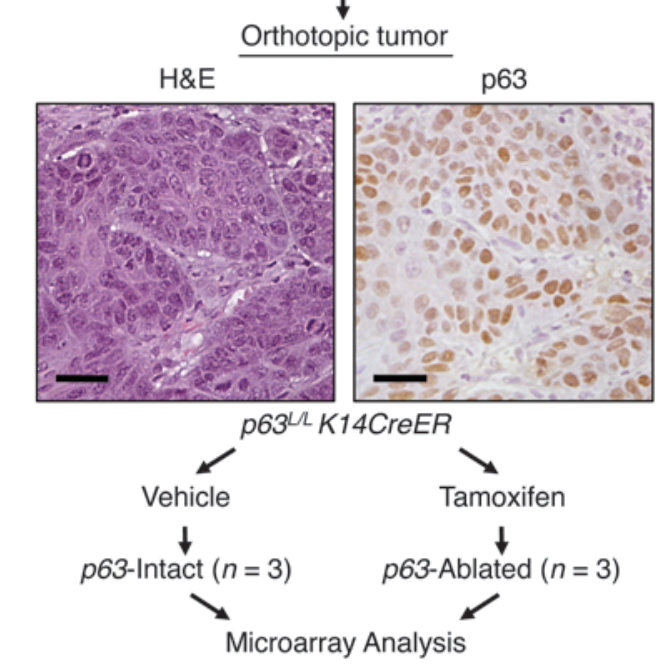

B

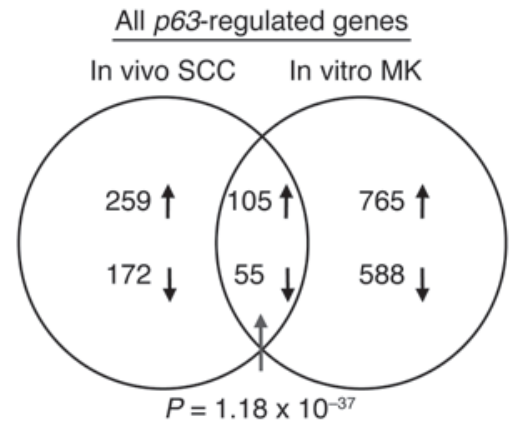

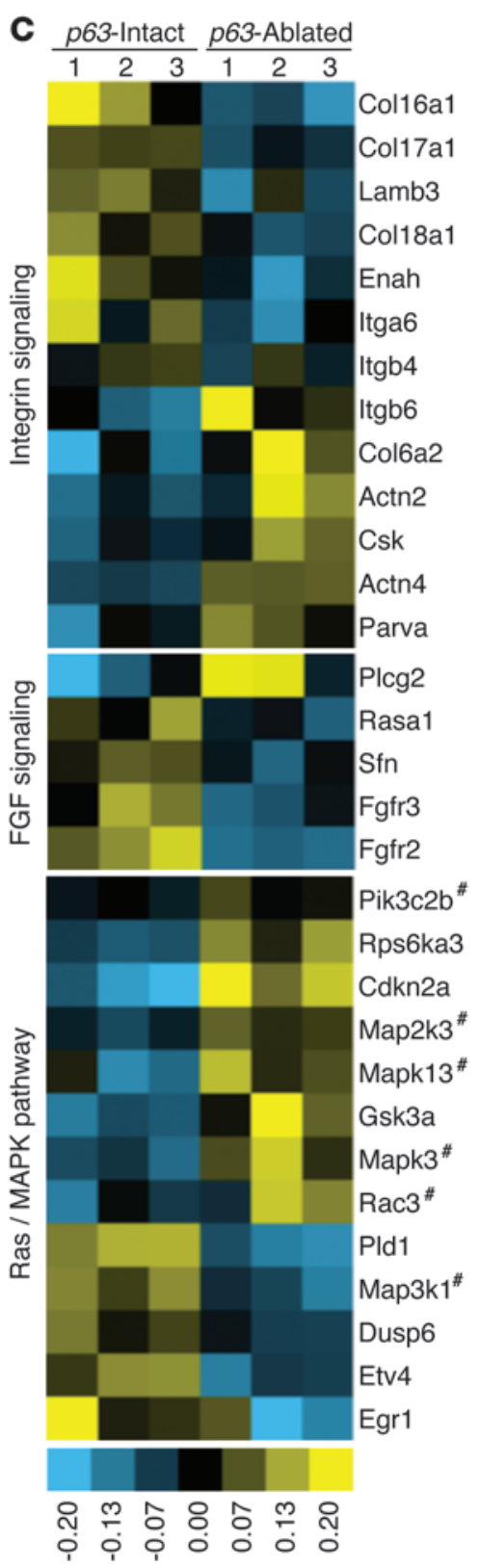

Figure 3

Comprehensive analysis of $p 63$-regulated transcription in SCC in vivo. (A) Schematic of orthotopic tumor generation for microarray analysis. Representative histology (H\&E) and p63 IHC staining of a primary tumor and its derivative orthotopic tumor are shown. Scale bars: $25 \mu \mathrm{m}$. (B) Overlap between p63-regulated genes identified in murine SCC tumors and previously identified $p 63$-regulated genes in cultured MK (81). $P$ value for overlap assessed by hypergeometric test. (C) Heat map of integrin signaling, FGF signaling, and RAS/MAPK signaling genes identified by DAVID analysis (39) as differentially expressed between p63intact and p63-ablated tumors. The $p 63^{L / L}$ K14-CreER consists of vehicle-treated $p 63$-ablated $(n=3)$, and $p 63$-ablated group consists of tamoxifen-treated $p 63^{L / L}$ K14-CreER $(n=3)$. Hatch marks indicate genes shared by all 3 pathways.
mRNA between 50- and 200-fold greater than TAp63 (Figure 1F), consistent with similar findings observed in human HNSCC cell lines (18). Taken together, these data demonstrate that this model shares key molecular and histologic features of human SCC.

Exquisite sensitivity of autochthonous SCC to loss of $p 63$. To determine whether tumors were dependent on p63, we employed a conditional $p 63$ allele $\left(p 63^{L / L}\right)$ (Supplemental Figure 2A). A previous study showed that Cre-mediated recombination in homozygous $p 63^{L / L}$ mice during embryogenesis recapitulates both the genomic structure and the phenotype of the p63-null mouse (34). In order to control $p 63$ excision in a tissue- and temporal-specific manner, we crossed these mice to a transgenic strain expressing the Cre recombinase/estrogen receptor (CreER) fusion protein from the keratin 14 (K14) promoter (35). Notably, our murine tumors demonstrate high overlap between p63 and K14 expression (Figure 2A), as was previously shown in human SCC (36). Given the importance of p53 in human SCC, we then performed additional crosses to generate $p 63^{L / L} \mathrm{~K} 14$-CreER mice on a $p 53^{+/-}$background for DMBA treatment. Mice were administered DMBA on the back skin or in the oral cavity until tumors reached approximately $100 \mathrm{~mm}^{3}$, a size not reached by papillomas (Figure 1A), then treated for 5 consecutive days with $100 \mathrm{mg} / \mathrm{kg}$ tamoxifen. Allele-specific PCR (Supplemental Figure 2A) demonstrated that p63 was excised only in tissues that normally express K14 (Figure 2B and ref. 37). Immunohistochemical (IHC) staining 72 hours after tamoxifen treatment confirmed that p63 is rapidly and efficiently ablated from established SCC tumors in a temporally controlled manner (Figure 2C).

A tumor-bearing cohort of $p 63^{L / L}$ K14-CreER mice served as the experimental arm, while tumor-bearing cohorts of $p 63^{L / L}$, $p 63^{+/ L}$, and $p 63^{+/ L} \mathrm{~K} 14$-CreER mice served as controls. All tumors of control mice showed progressive growth following tamoxifen 
Table 1

p63-regulated pathways in murine SCC assessed by DAVID

\begin{tabular}{|c|c|c|c|}
\hline Category & Term & Count & $P$ value \\
\hline KEGG_PATHWAY & mmu05200:Pathways in cancer & 23 & $7.36 \times 10^{-4}$ \\
\hline KEGG_PATHWAY & mmu05212:Pancreatic cancer & 8 & $8.60 \times 10^{-3}$ \\
\hline KEGG_PATHWAY & mmu04810:Regulation of actin cytoskeleton & 14 & $2.43 \times 10^{-2}$ \\
\hline KEGG_PATHWAY & mmu04010:MAPK signaling pathway & 16 & $2.55 \times 10^{-2}$ \\
\hline KEGG_PATHWAY & mmu04340:Hedgehog signaling pathway & 6 & $3.03 \times 10^{-2}$ \\
\hline KEGG_PATHWAY & mmu04144:Endocytosis & 13 & $3.16 \times 10^{-2}$ \\
\hline KEGG_PATHWAY & mmu04370:VEGF signaling pathway & 7 & $3.67 \times 10^{-2}$ \\
\hline KEGG_PATHWAY & mmu04670:Leukocyte transendothelial migration & 9 & $3.97 \times 10^{-2}$ \\
\hline KEGG_PATHWAY & mmu04666:Fc gamma R-mediated phagocytosis & 8 & $4.00 \times 10^{-2}$ \\
\hline KEGG_PATHWAY & mmu04960:Aldosterone-regulated sodium reabsorption & 5 & $4.67 \times 10^{-2}$ \\
\hline KEGG_PATHWAY & mmu05219:Bladder cancer & 5 & $4.67 \times 10^{-2}$ \\
\hline PANTHER_PATHWAY & P04393:Ras Pathway & 10 & $4.87 \times 10^{-3}$ \\
\hline PANTHER_PATHWAY & P00034:Integrin signaling pathway & 17 & $9.26 \times 10^{-3}$ \\
\hline PANTHER_PATHWAY & P00021:FGF signaling pathway & 11 & $2.15 \times 10^{-2}$ \\
\hline GOTERM_BP_FAT & G0:0060429 epithelium development & 25 & $5.07 \times 10^{-6}$ \\
\hline GOTERM_BP_FAT & G0:0048732 gland development & 20 & $1.50 \times 10^{-5}$ \\
\hline GOTERM_BP_FAT & G0:0030879 mammary gland development & 13 & $1.52 \times 10^{-5}$ \\
\hline GOTERM_BP_FAT & G0:0035239 tube morphogenesis & 18 & $2.84 \times 10^{-5}$ \\
\hline GOTERM_BP_FAT & G0:0035295 tube development & 22 & $9.37 \times 10^{-5}$ \\
\hline GOTERM_BP_FAT & G0:0030855 epithelial cell differentiation & 14 & $1.32 \times 10^{-4}$ \\
\hline GOTERM_BP_FAT & G0:0048729 tissue morphogenesis & 20 & $1.93 \times 10^{-4}$ \\
\hline GOTERM_BP_FAT & GO:0022612 gland morphogenesis & 11 & $2.98 \times 10^{-4}$ \\
\hline GOTERM_MF_FAT & G0:0003779 actin binding & 22 & $4.02 \times 10^{-4}$ \\
\hline GOTERM_BP_FAT & G0:0060443 mammary gland morphogenesis & 7 & $5.07 \times 10^{-4}$ \\
\hline GOTERM_BP_FAT & G0:0008544 epidermis development & 13 & $5.79 \times 10^{-4}$ \\
\hline GOTERM_BP_FAT & G0:0001763 morphogenesis of a branching structure & 13 & $5.79 \times 10^{-4}$ \\
\hline GOTERM_MF_FAT & G0:0005198 structural molecule activity & 29 & $6.42 \times 10^{-4}$ \\
\hline GOTERM_BP_FAT & GO:0030036 actin cytoskeleton organization & 15 & $7.29 \times 10^{-4}$ \\
\hline GOTERM_BP_FAT & G0:0060562 epithelial tube morphogenesis & 12 & $7.44 \times 10^{-4}$ \\
\hline GOTERM_CC_FAT & G0:0005886 plasma membrane & 122 & $8.55 \times 10^{-4}$ \\
\hline GOTERM_BP_FAT & G0:0001666 response to hypoxia & 9 & $8.57 \times 10^{-4}$ \\
\hline GOTERM_MF_FAT & G0:0008092 cytoskeletal protein binding & 27 & $8.69 \times 10^{-4}$ \\
\hline GOTERM_BP_FAT & G0:0070482 response to oxygen levels & 9 & $9.50 \times 10^{-4}$ \\
\hline GOTERM_BP_FAT & G0:0007398 ectoderm development & 13 & $1.01 \times 10^{-3}$ \\
\hline
\end{tabular}

performed global gene expression profiling following p63 genomic excision using an orthotopic primary tumor transplant system we developed (Figure 3A and ref. 25). Importantly these tumors can be serially transplanted (Supplemental Figure 3A) and grow with rapid kinetics (Supplemental Figure 3, $\mathrm{B}$ and $\mathrm{C}$ ). This system therefore allows us to generate multiple tumors with the same genetic alterations and endogenous tumor stroma, all growing in vivo at the same time and at the same rate in multiple mice. We can thus conduct carefully controlled replicate in vivo experiments. Notably, these tumors share the same histological features and p63 expression as the primary tumor (Figure 3A). Similar to autochthonous tumors (Figure 2D), only $p 63^{L / L} \mathrm{~K} 14$-CreER tumors and not $p 63^{\mathrm{L} / \mathrm{L}}$ tumors had reduced growth following tamoxifen treatment (Supplemental Figure 3, B and $\mathrm{C}$ ). We then collected whole tumors at multiple defined intervals following treatment, prepared RNA, and performed global gene expression profiling using Illumina MouseWG-6 BeadChip arrays. Results were analyzed for significant differences between $p 63^{L / L}$ K14-CreER tumors treated with vehicle (hereafter $p 63$-intact) and tamoxifen (hereafter $p 63$-ablated),

treatment (Figure 2D). In contrast, all $p 63^{L / L}$ K14-CreER tumors showed rapid and dramatic regression within a few days of $p 63$ excision, which was observed in both cutaneous (Figure 2D and Supplemental Figure 2B) and oral SCCs (Supplemental Figure 2C). Importantly, we saw no evidence of effects on normal epithelial tissues, consistent with the reported effects of $p 63$ loss in the normal epidermis that appear only after a period of months (34). Histological analysis after the last dose of tamoxifen demonstrated apoptotic features in $p 63^{L / L} \mathrm{~K} 14$-CreER tumors compared with control $p 63^{L / L}$ tumors (Figure $2 \mathrm{E}$ ). Staining for cleaved caspase 3 by IHC confirmed the marked induction of apoptosis in these tumors (Figure 2, E and F). In addition, Ki67 staining demonstrated a significant decrease in proliferation in the tamoxifen-treated $p 63^{L / L}$ K14-CreER tumors compared with controls, which became more pronounced at later time points after excision (Figure 2, E and G). Taken together, these data demonstrate exquisite dependence of endogenous SCC on p63 for survival and proliferation.

A central role for p63-regulated developmental signaling in endogenous SCC. It is unknown whether any of the established transcriptional targets or functions of p63 are critical for maintenance of SCC in vivo. In order to systematically identify such key programs, we revealing 834 transcripts that had a greater than 1.5 -fold change and a $P$ value of less than 0.05 following $p 63$ excision.

The physiologic relevance of this $p 63$-ablated versus $p 63$-intact gene expression data set is supported by the significant overlap we observed between genes changed following p63 excision in our SCC tumors (Figure $3 \mathrm{~B}$ ) and those regulated in normal murine keratinocytes (MK) following p63-directed siRNA (38). We then used the Database for Annotation, Visualization, and Integrated Discovery (DAVID) (39) tool to carry out pathway analysis on these genes. Indeed, Gene Ontogeny (GO) analysis revealed "epithelium development" and "epithelial cell differentiation" as 2 of the most significant GO terms for both SCC and MK gene sets and for the set of overlapping genes (Table 1). This finding suggests that deregulation of its normal developmental programs may contribute to p63-dependent tumor maintenance. Consistent with this possibility, pathway analysis identified genes involved in integrin signaling (e.g., Col6 $\alpha 2$, Col17 $\alpha 1, \operatorname{Lam} \beta 3$ ), an important p63-regulated developmental program $(9,15,40)$, as enriched following $p 63$ excision in SCC (Table 1 and Figure 3C). This analysis also uncovered a functional signature associated with Ras/MAPK signaling, involving genes including Dusp6, Egr1, and Etv4, and Pld1 (Table 1 and Figure 3C). Notably, this transcriptional program has not 


\section{A Orthotopic SCC}

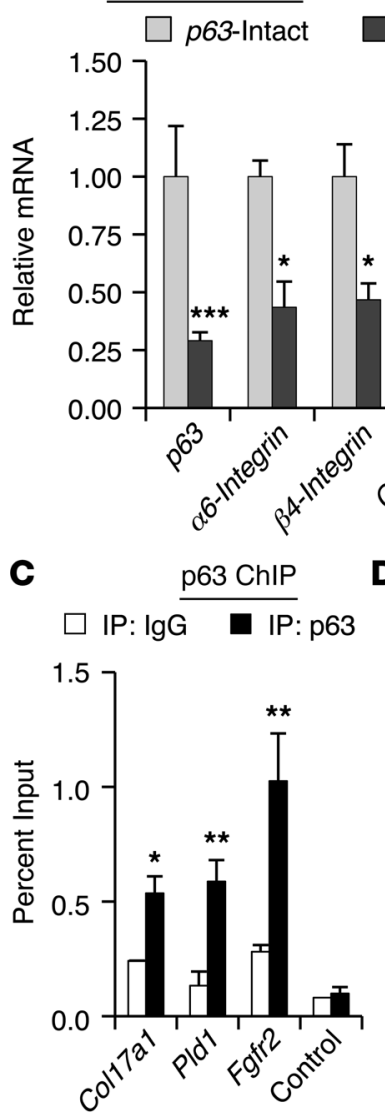

G

$\underline{\text { Soft agar colonies }}$ (FaDU cells)

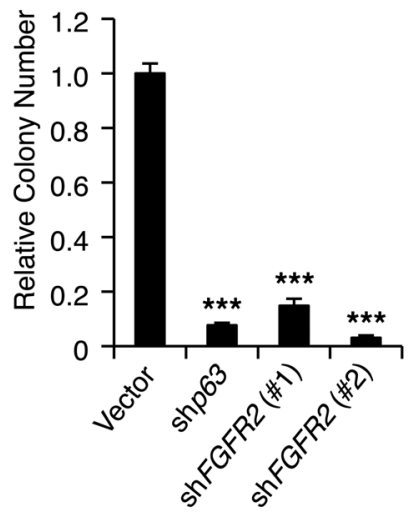

p63-Ablated

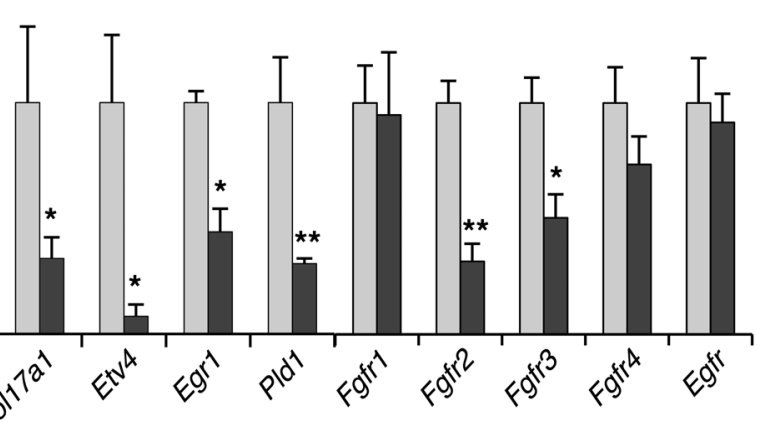

B

\section{Autochthonous SCC}

FGFR2

p63

S6

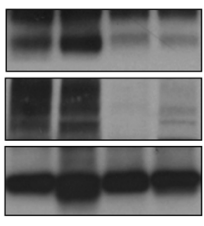

$\begin{array}{lllll}\text { Treatment } & \mathrm{T} & \mathrm{T} & \mathrm{T} & \mathrm{T}\end{array}$

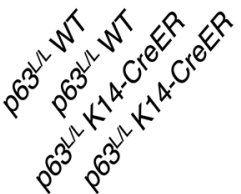

$\mathbf{F}$

E

Autochthonous SCC

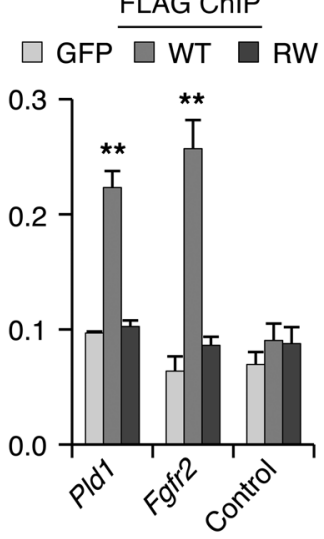

Fgfr2 IIlb $\square$ Fgfr2 IIlc $\square$ Fgfr3
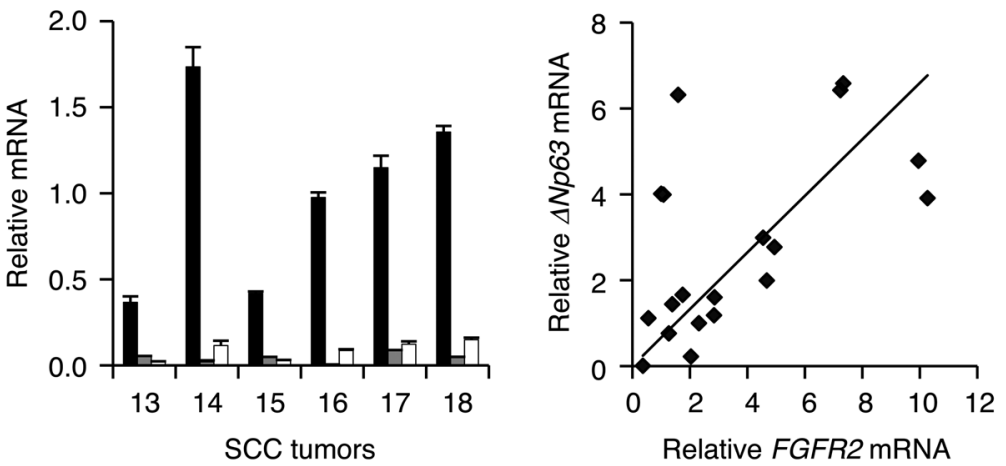

H

FaDU human HNSCC
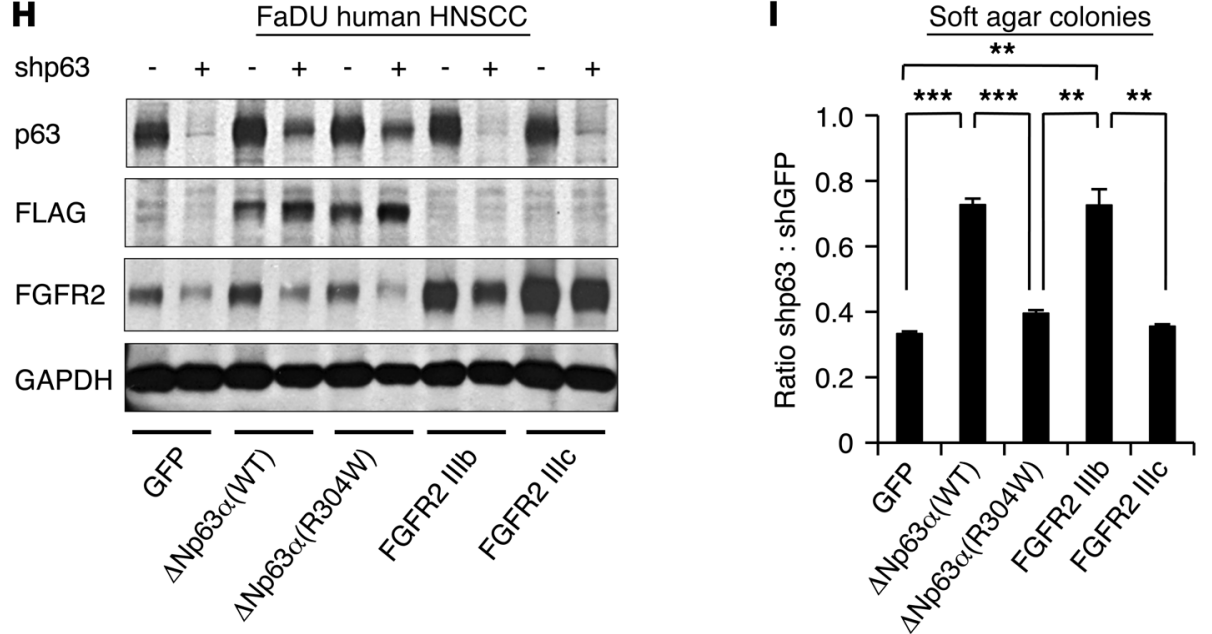

Figure 4

Direct regulation and requirement for p63-induced Fgfr2 in SCC. (A) QRT-PCR assessment of mRNA levels of indicated genes in $p 63-$ intact $(n=3-5)$ or p63-excised $(n=3-4)$ orthotopic tumor samples normalized to $\beta$-actin. (B) Western blot of indicated proteins in SCC tumors of indicated genotype. Ribosomal S6 serves as a loading control. T, tamoxifen. (C) ChIP of endogenous p63 at the Col17 $\alpha 1$, Pld1, and Fgfr2 loci in murine B9 SCC cells using indicated antibodies. Control designates highly conserved region $-2.4 \mathrm{~kb}$ upstream of Fgfr2 promoter. (D) ChIP of exogenously expressed, FLAG-epitope tagged WT $\Delta$ Np63 $\alpha$ or DNA-binding-deficient $\Delta$ Np63 $\alpha$ (R304W) in B9 cells. GFP-expressing cells serve as a negative control. (E) Relative Fgfr mRNA levels in autochthonous murine tumors, normalized to $\beta$-actin. (F) Correlation between $\triangle N p 63$ and FGFR2 mRNA levels in human primary HNSCC tumors as assessed by TaqMan QRT-PCR. $P$ value was calculated using Pearson product-moment correlation coefficient. (G) Human FaDU HNSCC cells plated in soft agar 72 hours after infection with p63 or FGFR2-directed shRNAs. (H) Western blot analysis of FaDU HNSCC cells stably expressing indicated proteins 72 hours following infection with p63-directed shRNA or control shRNA. Exogenous $\triangle$ Np63 $\alpha$ (WT) and $\triangle N p 63 \alpha(R 304 \mathrm{~W})$ contain a FLAG epitope tag and are resistant to p63 shRNA (47). GAPDH serves as a loading control. (I) Colony forming assay of samples in $\mathbf{H} 10$ days after infection with $p 63$-directed or control shRNA. ${ }^{*} P<0.05 ;{ }^{* \star} P<0.01$; ${ }^{* * \star} P<0.001$ as assessed by Student's unpaired $t$ test for all experiments except where indicated. Error bars represent SEM. 
been previously ascribed to p63, but may contribute to p63 function in SCC, given that each of these genes has functional links to RAS/MAPK pathway activation in cancer $(41,42)$.

A third p63-dependent transcriptional program emerging from this analysis involved genes associated with FGF signaling, including Fgfr2 and Fgfr3 (Table 1 and Figure 3C). FGF signaling seemed to be a particularly noteworthy and potentially important pathway controlled by $\mathrm{p} 63$ in SCC for several reasons. First, this signaling pathway and these factors in particular are likely to be relevant in p63-expressing cells, as Fgfr2 $\mathrm{IIJb}^{-/}$mice bear striking similarities to $p 63^{-/-}$mice, including dramatic failure of limb and skin development $(14,43)$. Indeed, both FGFR2 and FGFR3 expression have been linked to $\mathrm{p} 63$ in a developmental context $(40,44)$. Second, we discovered through analysis of public data sets that FGFR2 is highly significantly overexpressed together with p63 in human SCC tumors versus normal epithelia (Supplemental Figure 3, D and E). Third, although neither FGFR2 nor FGFR3 has previously been associated with p63 in human cancer, both genes encode bona fide oncogenes and are subject to activating somatic alterations in human tumors $(45,46)$. Finally, there is significant overlap among all 3 of the p63-regulated gene sets we identified (integrin signaling, RAS/MAPK activation, FGF signaling), suggesting that these programs may converge on a common program that is essential for tumor maintenance (Figure 3C).

Direct p63-mediated regulation and requirement for FGFR2 signaling in $S C C$. We next validated the microarray results, using quantitative RT-PCR (QRT-PCR) to confirm reduced mRNA levels of Fgfr2/3, the adhesion- and RAS/MAPK-associated genes in p63-ablated tumors compared with $p 63$-intact tumors (Figure 4A). In contrast, we saw no significant changes in control genes, including Fgfr1, Fgfr4, or Egfr, following inactivation of p63 (Figure 4A). Reduction in Col17 1 1, Pld1, Fgfr2, and Fgfr3 mRNA was further confirmed in primary autochthonous murine SCC tumors (Supplemental Figure 4A), and reduction of the corresponding proteins was confirmed by Western analysis (Figure 4B). We then wished to determine whether the p63-regulated genes were subject to direct transcriptional control by $\mathrm{p} 63$ in SCC, since neither Col17 $\alpha 1$ nor Pld1 had previously been identified as p63-regulated genes. We performed ChIP using $\alpha$-p63 antibodies in murine B9 SCC cells (Supplemental Figure 4B) and observed significant p63 binding upstream of the Col17 $\alpha 1$ and $F g f r 2$ transcription start sites and in the first intron of Pld1 (Figure 4C). To confirm the specificity of these findings, we expressed FLAG-epitope-tagged WT $\Delta \mathrm{Np} 63 \alpha$ (WT) or a DNA-binding deficient point mutant $\Delta \mathrm{Np} 63 \alpha$ (R304W) (47) in B9 cells and performed ChIP using antibodies against the FLAG epitope (Supplemental Figure 4B). Only cells expressing WT $\Delta \mathrm{Np} 63 \alpha$ demonstrated significant binding of $\Delta \mathrm{Np} 63 \alpha$ to the Pld 1 and Fgfr2 loci (Figure 4D). Collectively, these data identify Col17 $\alpha 1$, Pld1, and Fgfr2 as direct, p63-activated target genes in SCC.

The Fgfr 2 transcript undergoes alternative splicing, resulting in an Fgfr2 IIIb isoform, preferentially expressed on epithelial cells, and an Fgfr2 IIIc isoform expressed on mesenchymal cells (48). Examination of mRNA levels in autochthonous SCC tumors (Figure 4E) showed that the epithelial-specific Fgfr2 IIIb isoform was expressed at substantially higher levels than Fgfr2 IIIC or Fgfr3, suggesting that Fgfr2 IIIb is the most relevant p63-regulated isoform in SCC. In order to establish the relevance of FGFR2 in human tumors, we first examined the regulation of FGFR2 by p63 in HNSCC cell lines. As was seen in murine tumors, lentiviral-mediated knockdown of p 63 resulted in substantially reduced FGFR2 levels in human HNSCC cells (Supplemental Figure 4C). Next, we obtained primary human HNSCC tumor samples and examined the relationship between $\triangle N p 63$ and FGFR2 expression. Using QRT-PCR we found that FGFR2 and $\triangle N p 63$ mRNA levels were directly and highly statistically significantly correlated in these tumors (Figure 4F), consistent with a direct regulatory relationship.

We then wished to test the functional contribution of the p63-FGFR2 axis in SCC. Significantly, we found that lentiviral knockdown of either p63 or FGFR2 in human HNSCC cells was associated with similar dramatic suppression of anchorage-independent growth (Figure 4G) and colony forming ability (Supplemental Figure 4D). To determine whether $\mathrm{p} 63$ is sufficient to induce FGFR2 expression in this setting, we expressed either a WT $\Delta \mathrm{Np} 63 \alpha$ isoform, the DNA-binding-deficient mutant $\Delta \mathrm{Np} 63 \alpha$ (R304W), or TAp $63 \alpha$ (WT) in HaCAT-immortalized human keratinocytes. Only WT $\Delta \mathrm{Np} 63 \alpha$ induced significant expression of FGFR2 (Supplemental Figure $4 \mathrm{E}$ ), suggesting that $\Delta \mathrm{Np} 63 \alpha$ alone can activate FGFR2. To directly test the requirement for FGFR2 signaling in p63-dependent tumor maintenance, we performed a genetic rescue experiment in human FaDU HNSCC cells. We stably expressed either a control GFP protein or an shRNA-resistant FLAG-epitope tagged $\Delta \mathrm{Np} 63 \alpha(\mathrm{WT})$ or $\Delta \mathrm{Np} 63 \alpha(\mathrm{R} 304 \mathrm{~W})(47)$, followed by lentiviralmediated knockdown of endogenous p63 (Figure 4H). Expression of $\Delta \mathrm{Np} 63 \alpha$ (WT) was able to significantly rescue the decreased soft agar colony formation following p63 knockdown, while the DNAbinding-deficient $\Delta \mathrm{Np} 63 \alpha(\mathrm{R} 304 \mathrm{~W})$ was not (Figure $4 \mathrm{I})$. Most remarkably, overexpression of the IIIb isoform of FGFR2, but not the IIIc isoform, was able to rescue colony formation to the same extent as $\Delta \mathrm{Np} 63 \alpha$ itself (Figure $4 \mathrm{I}$ ). Collectively, these data suggest that FGFR2 IIIb is a key activated target of p63 in SCC, and they demonstrate the essential role of p63-dependent FGFR2 IIIb signaling in both human and murine SCC.

A paracrine FGF7-FGFR2 signaling axis is upregulated in autochthonous tumors. We noted substantially increased expression of both $p 63$ and its target FGFR2 in murine (Figure 5A) and human (Supplemental Figure 5A) autochthonous tumors relative to multiple SCC cell lines, leading us to hypothesize that the p63-dependent FGFR2 signaling axis might be more highly activated in tumors as compared with cultured cells. Indeed, immunofluorescent (IF) staining of primary tumors, using K14 as an epithelial cell marker and vimentin as a stromal marker, demonstrated dramatic membrane FGFR2 staining and nuclear p63 staining selectively within malignant epithelial cells (Figure 5B). The ligands for the epithelial-expressed FGFR2 IIIb include FGF1 and FGF7 and are typically produced by mesenchymal cells during normal development, resulting in paracrine signaling $(49,50)$. Consistent with the hypothesis that stromal-derived ligands signal in a paracrine fashion to FGFR2 IIIb in autochthonous SCC, IF showed that tumor cells were surrounded by a dense vimentinpositive stroma (Figure 5B). The tumor stroma appeared to comprise activated fibroblasts, as evidenced by SMA staining (Supplemental Figure 5B). Furthermore, we documented highly elevated expression of multiple FGFR2 ligands, including FGF1, FGF7, and FGF10 in whole tumors compared with cultured cell lines, with FGF1 and FGF7 being the most highly expressed (Figure 5C and Supplemental Figure 5C). We then examined expression of $\mathrm{Fg} f 7$, the most specific of the ligands for FGFR2 IIIb, and $p 63$ in FACS-isolated cell populations from autochthonous tumors, comparing CD49f ( $\alpha 6$-integrin) positive (tumor) versus negative (stromal) cells following exclusion of hematopoietic and endothelial cells. We observed robust expression 

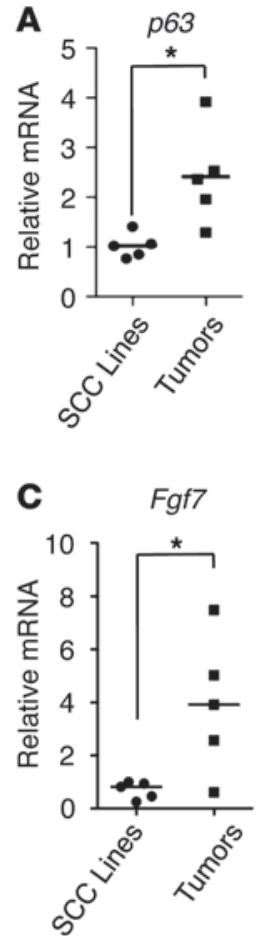
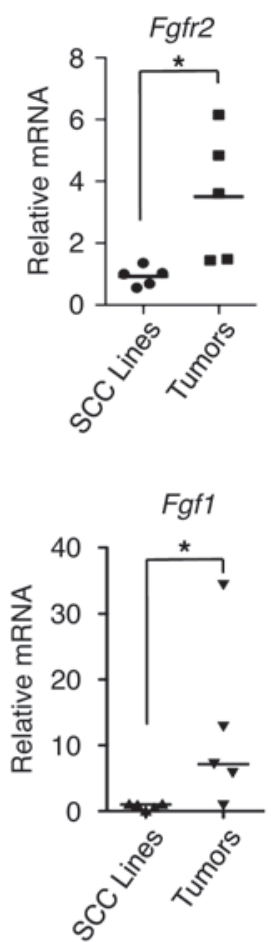

B
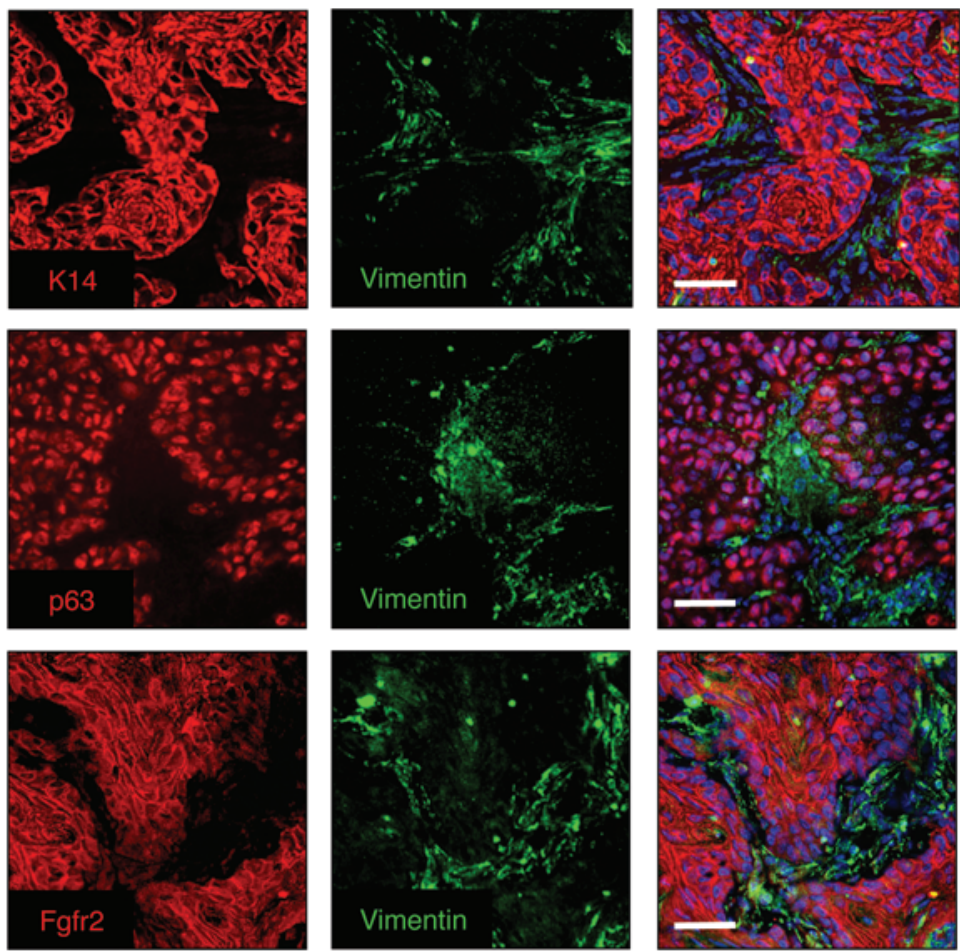

E
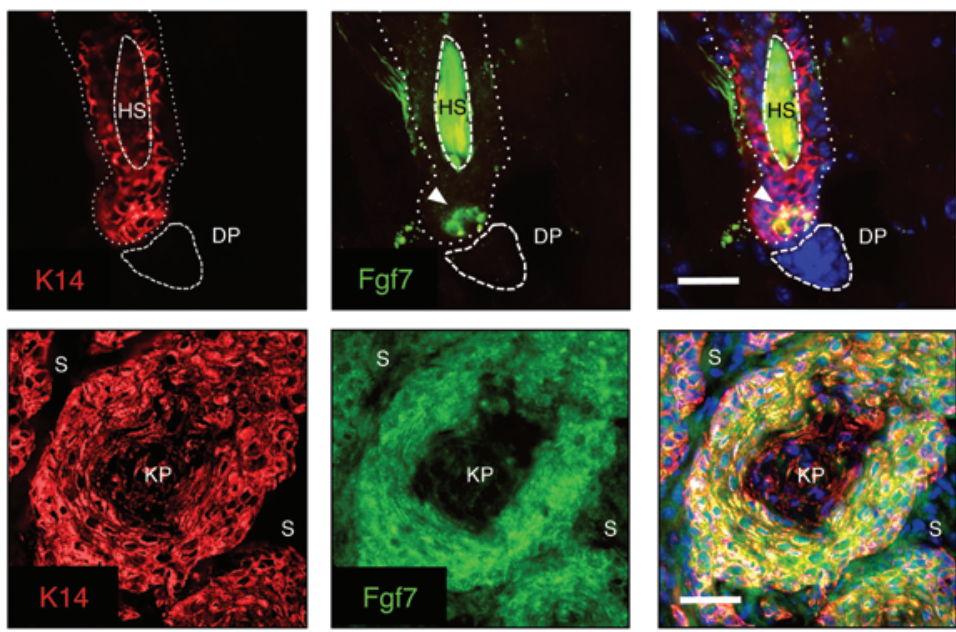

Figure 5

Deregulation of a paracrine FGFR2 signaling axis in autochthonous SCC. (A) Upregulation of p63 (left) and FGFR2 (right) in SCC tumors versus cell lines. Relative mRNA expression was determined by QRT-PCR in murine SCC cell lines $(n=5)$ and tumors $(n=5)$. Bar indicates mean value. ${ }^{*} P<0.05$ as assessed by Student's unpaired $t$ test. (B) IF staining of autochthonous murine SCC tumors, showing colocalization of K14, nuclear p63, and membrane-associated FGFR2. Vimentin (green) identifies stromal cells. Hoechst dye (blue) identifies nuclei. Scale bars: $25 \mu \mathrm{m}$. (C) Upregulation of FGFR2 ligands in SCC tumors. Relative mRNA expression of Fgf7 (left) and Fgf1 (right) in murine SCC cell lines $(n=5)$ and tumors $(n=5)$. Bars indicate mean value. ${ }^{*} P<0.05$ as assessed by Student's unpaired $t$ test. (D) Stroma-specific expression of FGF7 in autochthonous SCC. Primary tumors were disaggregated, then separated by FACS into epithelial CD49fhi ( $\alpha-6$ integrin) and stromal $\left(C D 49 f^{\circ}\right)$ populations following elimination of hematopoietic $\left(C D 45^{+}\right)$and endothelial $\left(C D 31^{+}\right)$cells prior to RNA analysis. Note epithelial-specific expression of p63. Error bars indicate SEM. ${ }^{* \star} P<0.01,{ }^{* \star \star} P<0.001$. (E) Spatially restricted FGF7 engagement in normal epithelia is abolished in SCC. IF staining of murine hair follicle in telogen (top) and autochthonous murine SCC tumors (bottom) for FGF7. K14 staining identifies epithelial cells. Note highly restricted expression in hair follicle (arrow) compared with tumor. HS, hair shaft; DP, dermal papilla; KP, keratin pearl; S, stromal cells. Scale bars: $25 \mu \mathrm{m}$. 
A
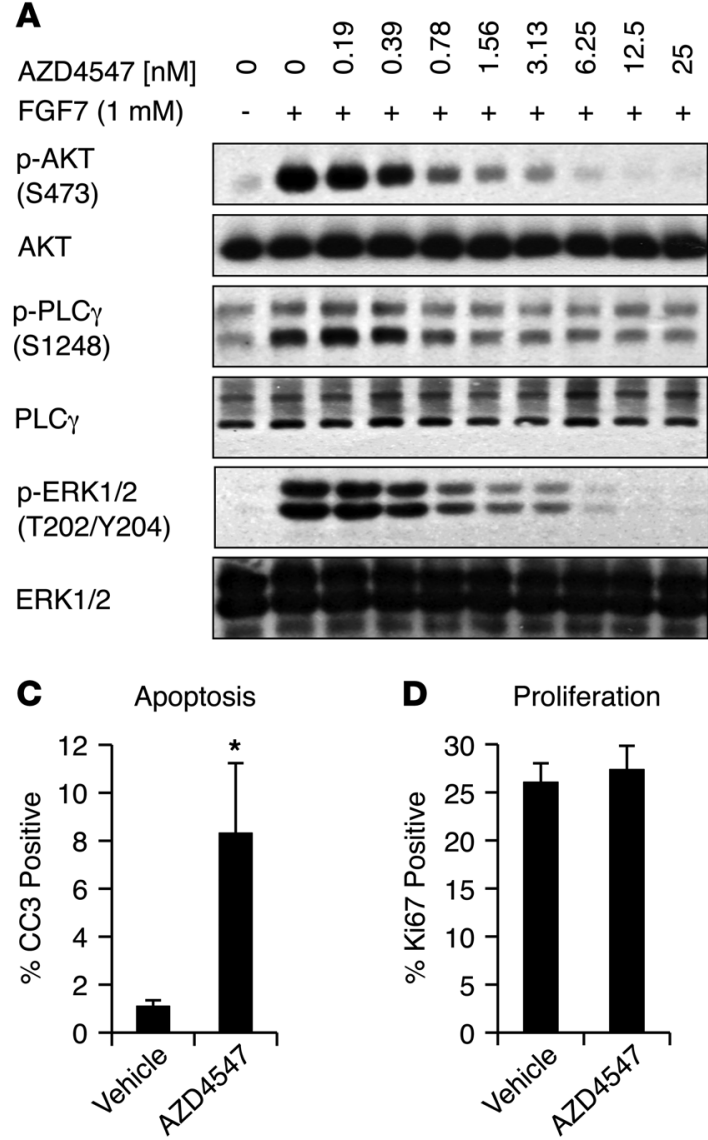

D Proliferation

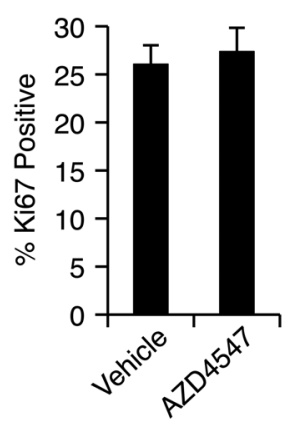

B

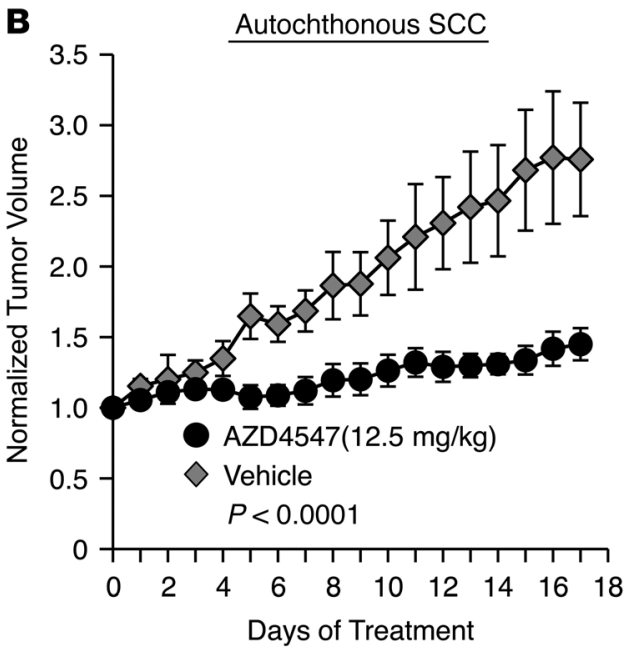

E

p-Akt

(S473)

Akt

p-PLC $\gamma$

(S1248)

PLC $\gamma$

p-Erk $1 / 2$

(T202/Y204)

Erk

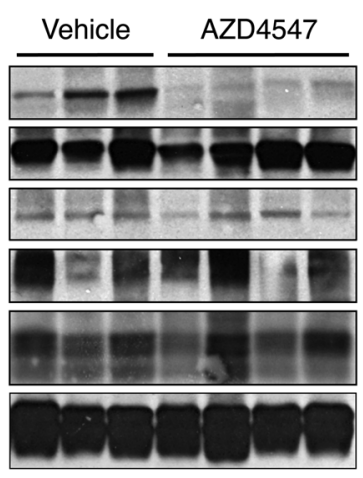

Figure 6

Therapeutic inhibition of ligand-activated FGFR2 signaling in SCC. (A) Inhibition of ligand-dependent FGFR2 signaling with AZD4547 extinguishes AKT, ERK1/2, and PLC $\gamma$ phosphorylation. FaDU cells were serum starved and pretreated for 1 hour with the indicated doses of AZD4547, followed by a 15-minute stimulation with FGF7 ligand. (B) AZD4547 treatment blocks progression of autochthonous SCC. Mice bearing DMBAinduced SCC tumors received daily oral AZD4547 $(12.5 \mathrm{mg} / \mathrm{kg}, n=7)$ or vehicle control $(n=6)$. Error bars represent SEM. $P<0.0001$ as assessed by multiple-measures ANOVA. (C) AZD4547 treatment induces autochthonous tumor apoptosis in vivo. Tumors harvested after 17 days treatment as in B were assessed for cleaved caspase 3 staining by IHC. AZD4547 $(n=7)$, vehicle control $(n=5) .{ }^{*} P<0.05$ as determined by Mann-Whitney test. (D) Quantification of Ki67-positive cells in tumors as in C. AZD4547 $(n=7)$, vehicle control $(n=5)$. $P$ value not significant as determined by Student's unpaired $t$ test. (E) AZD4547 treatment inhibits PI3K signaling downstream of ligand-activated FGFR2 in SCC. Western blot analysis of autochthonous tumor lysates following 17 days of daily oral treatment with vehicle control or AZD4547 (12.5 mg/kg).

of Fgf7 in stromal cells and virtually no expression in tumor cells, while the reverse was true for $p 63$ (Figure 5D). Thus, FGFR2 and its ligand FGF7 are overexpressed in different cell populations in endogenous SCC, suggesting activated paracrine signaling in vivo.

In the normal adult epithelium (e.g., the regenerating hair follicle), FGF7 is produced by a select group of mesenchymal cells in the dermal papilla, resulting in restricted receptor engagement and limited signaling within the hair germ at the base of the follicle (51). We confirmed this finding by IF staining of murine skin, demonstrating that only a small fraction of epithelial cells at the hair follicle base are exposed to and uptake FGF7, whereas the majority of follicle epithelial cells (marked by K14) entirely lack FGF7 staining (Figure 5E and Supplemental Figure 5D). In dramatic contrast, we observed that the ligand-producing stroma surrounding the SCC cells in autochthonous tumors resulted in FGF7 engagement at high levels in essentially all tumor cells (Figure 5E). Of note, membrane FGFR2 staining appeared less intense in tumor cells adjacent to FGF7-releasing stroma, in keeping with receptor internalization following ligand engagement (Supplemental Figure 5E). Together, these findings uncover a prominent role for disorganized and deregulated paracrine signaling via p63induced FGFR2 in the pathogenesis of endogenous SCC.

Therapeutic inhibition of FGFR signaling induces apoptosis in autochthonous SCC. In order to directly validate our model of hyperactivated ligand-induced FGFR2 signaling in SCC, we tested the effect of pharmacologic FGFR2 inhibition in these tumors. Notably, as an established oncogenic tumor driver, FGFR2 represents a potentially attractive therapeutic target in human cancer (52). We obtained an orally bioavailable FGFR inhibitor, AZD4547, which exhibits high specificity for FGFR1, -2, and -3 and low nanomolar potency for FGFR2 inhibition (53). We first examined FGFR2 signaling in FaDU HNSCC cells, which exhibit FGFR2 levels approaching those we observed in vivo (Supplemental Figure 6A). Cells were serum starved, then stimulated with FGF7 in the presence of increasing doses of AZD4547. Like many growth factor receptor tyrosine kinases, FGFR2 is reported to signal through multiple 
downstream pathways, including MAPK, PI3K, and PLC $\gamma$ (52). Low nanomolar doses of AZD4547 potently abolished FGF7stimulated signaling to AKT and ERK, but were less effective at inhibiting signaling through other reported downstream pathways including p38 and STAT3 (Figure 6A and Supplemental Figure 6B). Inhibition by AZD4547 was specific for FGFR2-dependent signaling, as this compound did not block EGF-dependent signaling (Supplemental Figure 6B) and had similar effects on both human and murine SCC cell lines (Supplemental Figure 6C). These data demonstrate that AZD4547 potently and specifically blocks ligand-mediated FGFR2 signaling in SCC cells.

The rationale for therapeutic targeting of FGFR2 has focused largely on relatively uncommon tumors that exhibit activating somatic mutations resulting in ligand-independent signaling (45). Our findings suggest that FGFR2 upregulation and activated paracrine signaling in SCCs may confer pathway dependence in the absence of receptor mutation. To test this hypothesis, we established a cohort of mice bearing autochthonous DMBAinduced SCCs and we treated these mice with either $12.5 \mathrm{mg} / \mathrm{kg} / \mathrm{d}$ AZD4547 or control vehicle. This dose was well tolerated by mice, which exhibited no overt physiologic effects compared with vehicle-treated mice. Remarkably, however, AZD4547 treatment resulted in a virtual complete arrest of tumor progression, which was apparent as early as 5 days following treatment initiation and which was associated with a dramatic increase in progress-free survival of AZD4547-treated mice (Figure 6B and Supplemental Figure 6D). Analysis of tumors following AZD4547 treatment showed an increase in apoptosis as assessed by cleaved caspase 3 staining, while there was no discernible effect on proliferation (Figure 6, $\mathrm{C}$ and D). We then determined which of the potential FGFR2-activated signaling pathways was affected in vivo by AZD4547 treatment of autochthonous tumors. We observed suppression of AKT phosphorylation following AZD4547 treatment, but little or no change in phosphorylation of ERK1/2 and PLC $\gamma$ (Figure 6E). The dramatic suppression of Akt phosphorylation in all AZD4547treated compared with vehicle-treated tumors is keeping with the established role of the PI3K/AKT pathway in cell survival and supports an essential role for p63-induced FGFR2 in maintaining survival of autochthonous SCCs through this pathway in vivo.

\section{Discussion}

Although it has been known for several years that p63 is overexpressed in a large proportion of primary SCCs $(18,32,54)$, p63 is also a marker of basal epithelial cells whose expression might simply reflect the lineage of origin for these tumors. Here, we use a sophisticated genetic model to show definitively that autochthonous SCCs are dependent upon high levels of endogenous p63. Thus, deletion of $p 63$ induces rapid tumor regression without any appreciable effects on normal p63-expressing epithelia (Figure 2). This finding is in stark contrast to the long latency required for phenotypic changes seen in normal tissues following in vivo excision of $p 63$ in the epithelium (34). These results define SCCs as functionally "p63-addicted" and furthermore suggest that therapies targeting the $\mathrm{p} 63$ pathway may benefit from a favorable therapeutic window. Indeed, the lack of toxicity we observe upon inhibiting the p63-FGFR2 axis using AZ4547, despite its dramatic effect on tumors, underscores this point (Figure 6).

While our model involved deletion of all $\mathrm{p} 63$ isoforms, our findings support a predominant role for the $\Delta \mathrm{Np} 63 \alpha$ isoform in the observed effects. $\Delta \mathrm{Np} 63 \alpha$ is by far the major isoform expressed in these tumors, and it binds directly to a conserved regulatory region to induce expression of FGFR2, which we show to be a central mediator of p63-dependent tumor survival (Figure 4). In contrast, TAp63 $\alpha$ is unable to modulate expression of FGFR2 (Supplemental Figure 3). The particular contribution of $\Delta \mathrm{Np} 63 \alpha$ to squamous carcinogenesis is also supported by data presented here (Figure 4$)$ and previously $(10,47)$ showing that $\Delta \mathrm{Np} 63 \alpha$ expression can rescue cell death induced by endogenous p 63 knockdown and that this isoform can cooperate with oncogenic Ras to promote squamous tumorigenesis (55).

Genome-wide in vivo gene-expression profiling of tumors following $p 63$ excision revealed multiple p63-dependent pathways with a plausible role in tumor maintenance, including integrin and MAPK signaling, as well as direct p63-regulated genes functioning within these pathways, including Col17 $\alpha 1$ and Pld1, respectively (Figure 3).

The most remarkable finding in our analysis, however, is a prominent growth factor signaling program converging on FGFR2, which we show to be a key and direct transcriptional target of p 63 in these tumors. The relevance of the p63-FGFR2 axis in human SCC is further supported by the strong correlation we observe between p63 and FGFR2 expression in primary human HNSCC tumors (Figure 4). We provide direct evidence arguing that FGFR2 mediates effects of p63 in SCC, showing that ectopic FGFR2 expression is sufficient to rescue effects of $\mathrm{p} 63$ knockdown and that FGFR2 rescues as effectively as ectopic expression of $\Delta \mathrm{Np} 63 \alpha$ itself (Figure 4). Additionally, we demonstrate that both FGFR2 and its ligands are produced at high levels in primary human and mouse tumors. By sorting such tumors, we demonstrated that in vivo the FGFR2 ligand FGF7 is produced exclusively from the stroma, yielding a paracrine signaling axis that is not recapitulated in cultured tumor cells (56). Indeed, a subset of SCC cell lines adapted to grow in the absence of stroma is known to activate autocrine FGFR signaling, consistent with our finding that this signaling is an important survival mechanism in these cancers (57). Most notably, whereas the normal developmental pattern of FGFR2 signaling involves limited and spatially restricted ligand exposure (51), we show that in endogenous SCC, the FGFR2-expressing tumor cells are surrounded by ligand-producing stroma and as a result exhibit high levels of receptor-associated ligand expression (Figure 5).

Widespread activation of p63-induced FGFR2 by overexpressed stromal ligand, together with other stroma-associated effects (58), may well explain the poor prognosis associated with an abundant stromal component in human SCCs $(21,22)$. Other recent studies provide further evidence supporting our finding that stromalderived growth factors and FGF7 in particular contribute to SCC pathogenesis. Deregulated signaling in stroma cells within the dermis induces a field cancerization effect associated with increased secretion of growth factors including FGF7 from the stroma (59), and transgenic expression of FGF7 itself results in transformation of the epithelium $(60,61)$. We have also found that other FGFR2 ligands, including FGF1 and FGF10, are elevated in the tumor microenvironment, suggesting that multiple ligands could potentially contribute to tumor progression. Finally, in addition to quantitative changes in FGFR2 and FGF expression, deregulated receptor spatial orientation and stromal juxtaposition within depolarized, disordered epithelia are likely to play a role in heightened signaling through this pathway (62).

While EGFR has long been considered the most important therapeutic target in SCC, the relatively modest preclinical $(63,64)$ and clinical activity of EGFR inhibitors against most SCCs, including 
HSNCC, might argue for the presence of an additional or alternative RTK-dependent pathway driving these tumors (65-67). Much of the attention in therapeutic targeting of FGFR-dependent pathways in SCC and other cancer contexts has focused on the relatively uncommon tumors exhibiting either amplification or somatic mutation of these receptors $(68,69)$. Our study suggests, however, that high levels of 63 itself are sufficient to drive FGFR2 expression, signaling, and tumor cell dependence. In this regard, it is notable that the inhibition of tumor growth we observed following treatment of mice with AZD4547 is comparable to that observed using the same dose of this compound in a xenograft model that employed an FGFR-amplified cell line (53). Our data also indicate that a major impact of AZD4547 is to potently inhibit PI3K/AKT signaling. Consequently, this compound induced apoptosis when used to treat autochthonous tumors (Figure 6). This observation may have may have direct relevance regarding which patient populations may benefit from treatment with this drug. A small but significant fraction of HNSCCs harbor either mutations in the catalytic PI3K subunit PI3KCA $(2,3)$ or loss of PTEN (70) and thus may exhibit resistance to this compound due to constitutive PI3K activity. Given that AZD4547 and other potent FGFR inhibitors are now entering the clinic, it is important to define the specific clinical context for its application. More broadly, our identification of a p63-driven pathway for growth factor signaling highlights the potential for therapeutic targeting of oncogenic transcriptional programs, even while targeting the transcription factors themselves remains a challenge.

\section{Methods}

\section{Mice}

To induce epidermal SCC tumors, 6-week-old mice were shaved and $25 \mathrm{mg}$ DMBA (Sigma-Aldrich) dissolved in acetone was applied to the skin once per week. For oral SCC tumors, DMBA was applied to the inside of the oral cavity weekly. Tumors were measured daily, and tumor volumes were calculated using the following formula: tumor volume $\left(\mathrm{mm}^{3}\right)=4 / 3 \pi \times$ length $/ 2 \times$ width $/ 2$. In order to excise $p 63$, mice were treated once per day with $100 \mathrm{mg} / \mathrm{kg}$ tamoxifen (Sigma-Aldrich) dissolved in sunflower seed oil (Sigma-Aldrich) by intraperitoneal injection for 5 days. Orthotopic transplants were performed as described previously (25). For in vivo AZD4547 (AstraZeneca Pharmaceuticals LP) treatment, tumor-bearing mice were treated daily by oral gavage with $12.5 \mathrm{mg} / \mathrm{kg}$ AZD4547 dissolved in 1\% Tween-80. Generation of $p 53^{-/-}, p 63^{L / L}$ mice, and K14-CreER mice has been previously described (30, 35, 37). Analysis of genomic p63 excision by PCR has been previously described (34).

\section{AZD4547 treatment of cell lines}

Inhibition of signaling by AZD4547 was performed by plating cells in $0.1 \%$ FBS for 22 hours, then serum-free medium for 1 hour. Freshly made AZD4547 was dissolved in DMSO and added to the medium for 1 additional hour. Then, $1 \mu \mathrm{M}$ recombinant human FGF7 (Sigma-Aldrich) was added to cells for 15 minutes followed by lysis with RIPA buffer. IC S0 $_{50}$ values of SCC cells treated with AZD4547 were determined 72 hours after plating of 500 cells/ well in medium containing 10\% FBS using the CellTiter-Glo Luminescent Cell Viability Assay (Promega) according to the manufacturer's instructions.

\section{Human tumor analysis}

Primary HNSCC tumors used in this study have been previously described (25). Following pathological review, total RNA was extracted from macrodissected tumor-enriched portions. Quantification of mRNA from tumor samples was performed using the TaqMan (Applied Biosystems) primer- probe sets according to the manufacturer's protocol. $\beta$-Actin mRNA expression in tumors served as a normalization control. Primer and probe sets are described in Supplemental Table 1.

\section{IHC and IF}

Assistance in processing of murine tumor samples was provided by the Dana-Farber/Harvard Cancer Center Specialized Histopathology Core Facility. Five-micron sections were cut from formalin-fixed, paraffinembedded tumors and stained using standard protocols. Staining for cleaved caspase 3 was performed using the SignalStain Cleaved Caspase-3 (Asp175) IHC detection kit (Cell Signaling) according to the manufacturer's instructions. For IF staining, murine tumors were snap-frozen in HistoPrep (Fisher Scientific), and 5- $\mu \mathrm{M}$ sections were cut. Samples were fixed in $4 \%$ paraformaldehyde and permeabilized with $0.1 \%$ Triton X-100 or fixed and permeabilized with methanol (K14; SMA). Nuclei were visualized by staining with Hoechst 33342 dye (Invitrogen). Stains with the SMA antibody used the Mouse on Mouse (M.O.M.) Basic Kit (Vector Laboratories) according to the manufacturer's instructions.

\section{Cell lines}

Murine cell lines B9 (26) and SCC/80 (71) were gifts of Nabeel Bardeesy (Massachusetts General Hospital). Murine cell lines mSCC (25), 392-1, and 1459 A were generated from DMBA-induced tumors and established in R\&G medium (71). Following establishment of a cell line, cells were switched to RPMI medium. JHU-029 (18) cells were a gift of David Sidranski (Johns Hopkins University, Baltimore, Maryland, USA). FaDU (72) and HaCAT (73) cells were a gift of James Rocco (Massachusetts General Hospital). HO1N1 (74), BICR-78 (75), CAL-33 (76), KYSE-30, and KYSE-150 (77) were a gift of S. Michael Rothenberg (Massachusetts General Hospital). Cells were maintained at $37^{\circ} \mathrm{C}$ in $5 \% \mathrm{CO}_{2}$ in either RPMI or DMEM (HaCAT) supplemented with $10 \%$ FBS, penicillin, and streptomycin. All human cell lines were maintained by the MGH Center for Molecular Therapeutics cell bank and underwent high-density SNP typing to confirm their identity.

\section{Real-time PCR}

Isolated tumor cells from autochthonous tumors were generated by disaggregation of primary tumors with collagenase, and cells were FACS sorted for viable CD $31^{\text {neg }}, \mathrm{CD} 45^{\text {neg }}$, and CD49f hi cells. For all experiments, RNA was extracted from whole tumors using QIAGEN RNeasy Fibrous Tissue Mini Kit according to the manufacturer's instructions. QRT-PCR analysis was performed using IQ SYBR Green Supermix (Bio-Rad) according to the manufacturer's instructions. Primers used for QRT-PCR are listed in Supplemental Table 1.

\section{Western blotting and ChIP}

Protein was extracted from tumors by incubation for 1 hour at $4^{\circ} \mathrm{C}$ in RIPA buffer (10 mM Tris- $\mathrm{HCl}, \mathrm{pH} 7.5,150 \mathrm{mM} \mathrm{NaCl}, 1 \mathrm{mM}$ EDTA, 1\% [w/v] sodium deoxycholate, $0.1 \%[\mathrm{w} / \mathrm{v}] \mathrm{SDS}, 1 \%[\mathrm{v} / \mathrm{v}] \mathrm{NP}-40$, proteinase inhibitor cocktail, phosphatase inhibitor cocktail) following physical grinding. ChIP was performed as previously described (47). Data represent 3 to 5 independently immunoprecipitated samples per antibody. Primer sets used can be found in Supplemental Table 1.

\section{Lentiviral, retroviral production, colony forming assays, and $m R N A$ QRT-PCR}

Production of virus and mRNA analysis were performed as described previously (18). Soft agar colony-forming assays were performed by plating $3 \times 10^{4}$ cells per well in a 6 -well plate in $0.4 \%$ agarose 72 hours following infection with lentiviral shRNA constructs. Rescue experiments in Figure 4, H and I, were supplemented with $1 \mu \mathrm{M}$ FGF7. Data represent 
average of 3 random fields in 3 independently seeded wells, performed twice with similar results.

\section{Plasmids}

Retroviral pBABE (puro)-FGFR2 IIIb and pBABE (puro)-FGFR2 IIIc plasmids (45) were a gift of Heidi Greulich (Broad Institute, Cambridge, Massachusetts, USA). pLPC- $\Delta$ Np63 $\alpha$ (WT)-FLAG, $\Delta$ Np63 $\alpha$ (R304W)-FLAG, and pLPC-TAp63 $\alpha$ (WT)-FLAG plasmids have been previously described (47).

\section{Microarray}

RNA was extracted from whole orthotopic tumors using QIAGEN RNeasy Fibrous Tissue Mini Kit according to the manufacturer's instructions. Synthesis of cDNA, hybridization, and reading of signal intensity were performed by the Partners HealthCare Center for Personalized Genetic Medicine. RNA quality was assessed on a 2100 Bioanalyzer (Agilent), and total RNA was hybridized to Illumina MouseWG-6 v2 BeadChip arrays. Chips were scanned with Illumina BeadArray Reader to measure the signal intensity by the labeled target. Data were processed using the R statistical software environment, version 2.12.0 (78). Data were background corrected, normalized, and underwent variance stabilizing transformations using the Lumi package (79). Differential gene expression was determined based on a moderated $t$ test using the Limma package (80). The entire set of unprocessed raw data is available (GEO GSE45929).

\section{Quantification of proliferation and apoptosis following p63 excision}

For quantification of cleaved caspase 3 staining in Figure 2F, the day- 6 control group consisted of vehicle-treated $p 633^{L / L} \mathrm{~K} 14-\mathrm{CreER}(n=3)$ and tamoxifen-treated $p 63^{L / L}(n=2)$ mice compared with tamoxifen-treated $p 63^{L / L}$ K14-CreER mice $(n=5)$. The day 8 control group consisted of vehicle-treated $p 63^{L / L} \mathrm{~K} 14-\mathrm{CreER}(n=2)$ and tamoxifen-treated $p 63^{L / L}$ mice $(n=2)$ compared with tamoxifen-treated $p 63^{L / L} \mathrm{~K} 14$-CreER mice $(n=4)$. For quantification of Ki67 staining in Figure 2G, the day- 6 control group consisted of vehicle-treated $p 63^{L / L} \mathrm{~K} 14-\operatorname{CreER}(n=2)$ and tamoxifen-treated $p 63^{L / L}(n=1)$ compared with tamoxifen-treated $p 63^{L / L} \mathrm{~K} 14$-CreER mice $(n=4)$. The day- 8 control group consisted of vehicle-treated $p 63^{L / L} \mathrm{~K} 14$-CreER $(n=2)$ and tamoxifen-treated $p 63^{L / L}$ mice $(n=1)$ compared with tamoxifen-treated $p 63^{L / L} \mathrm{~K} 14$-CreER mice $(n=4)$.

\section{Antibodies}

Western blotting. PARP (Cell Signaling), GAPDH (FL335; Santa Cruz Biotechnology Inc.), p53 (DO-1; Santa Cruz Biotechnology Inc.), p63 (4A4; Sigma-Aldrich), BCL-2 (C2; Santa Cruz Biotechnology Inc.), Erk1/2 (K-23; Santa Cruz Biotechnology Inc.), FLAG (M2; Sigma-Aldrich), FGFR2 (Abcam), AKT (Cell Signaling), AKT-pSer473 (Cell Signaling), ERK1/2pThr202/Tyr204 (20G11; Cell Signaling), PLC $\gamma$ (D9H10; Cell Signaling), PLC $\gamma$-pSer1248 (Cell Signaling), p38-pThr180/Tyr182 (3D7; Cell Signaling), p38 (5F11; Cell Signaling), STAT3-pTyr705 (3E2; Cell Signaling), STAT3 (124H6; Cell Signaling), EGFR (1005, Santa Cruz Biotechnology Inc.), and EGFR-pTyr845 (Cell Signaling) were used.

ChIP. p63 $\alpha$ (H-129; Santa Cruz Biotechnology Inc.) and FLAG epitope (M2; Sigma-Aldrich) were used.

Immunohistochemistry. Ki67 (NCL-Ki67p; Novacastra), K14 (Covance), and $\mathrm{p} 63$ (4A4; Sigma-Aldrich) were used.

IF. FGF7 (H-73 or C-19; Santa Cruz Biotechnology Inc.), FGFR2 (ab52256; Abcam), p63 (H-129; Santa Cruz Biotechnology Inc.), Vimentin
(S-20; Santa Cruz Biotechnology Inc.), K14 (Covance), and $\alpha$-SMA (ab7817; Abcam) were used.

FACS. CD45 (biotin-conjugated; eBioscience), CD31 (biotin-conjugated; eBioscience), and CD49f (PE-conjugated; BD Biosciences Pharmingen) were used.

\section{Statistics}

All $P$ values were determined using the 2-tailed Student's unpaired $t$ test with the exceptions noted here. The values in Figure 2F and Figure 6C had non-Gaussian distributions and were analyzed using the Mann-Whitney test. For in vivo experiments in Figure 2D and Figure 6B, changes in tumor volume were analyzed using multiple-measures ANOVA. For Figure 4F, the Pearson product moment correlation coefficient $\left(R^{2}\right)$ was calculated, and the $P$ value was determined from a probability table. $P$ values for GO, KEGG, and Panther Pathways as analyzed using DAVID were determined using Fisher's exact test. $P<0.05$ was considered significant for all experiments.

\section{Study approval}

The Massachusetts General Hospital IRB approved use of tissues and clinical data for this study. All human tissue studies used exclusively discarded material collected in the course of routine clinical care, for which our IRB determined that signed informed consent was not required. Housing and treatment for all animals was approved by the Subcommittee on Research Animal Care at the Massachusetts General Hospital.

\section{Acknowledgments}

We wish to thank Nabeel Bardeesy, James Rocco, William Michaud, Mike Dennis, and Zach Nash for advice, reagents, and technical support; and Nabeel Bardeesy, Andrea McClatchey, and members of the Ellisen laboratory for comments on the manuscript. The Dana-Farber/Harvard Cancer Center Specialized Histopathology Core Facility assisted in processing and staining of murine tumors, and the Harvard Stem Cell Institute/Center for Regenerative Medicine (HSCI/CRM) Flow Cytometry Core Facility assisted with tumor sample sorting. AZD4547 used in this study was provided by AstraZeneca Pharmaceuticals LP. This work was supported by NIDCR R01 DE015945 (to L.W. Ellisen), by NCI K99 CA 157730 (to M.R. Ramsey), and by the American Cancer Society/Mass Biotech Council Cancer Research Challenge-AstraZeneca Pharmaceuticals LP Fellowship PF-09-100-01 MGO (to M.R. Ramsey).

Received for publication January 22, 2013, and accepted in revised form May 8, 2013.

Address correspondence to: Leif W. Ellisen, MGH Cancer Center, GRJ-904, 55 Fruit Street, Boston, Massachusetts 02114, USA. Phone: 617.726.4315; Fax: 617.726.8623; E-mail: ellisen@helix. mgh.harvard.edu.

Catherine Wilson's present address is: Discovery Oncology, Genentech, Inc., South San Francisco, California, USA.

Benjamin Ory's present address is: INSERM, UMR 957, Université de Nantes, Nantes, France.
1. Rothenberg SM, Ellisen LW. The molecular pathogenesis of head and neck squamous cell carcinoma. J Clin Invest. 2012;122(6):1951-1957.

2. Stransky N, et al. The mutational landscape of head and neck squamous cell carcinoma. Science. 2011; 333(6046):1157-1160.
3. Agrawal N, et al. Exome sequencing of head and neck squamous cell carcinoma reveals inactivating mutations in NOTCH1. Science. 2011; 333(6046):1154-1157.

4. Argiris A, et al. Phase III randomized, placebo-controlled trial of docetaxel with or without gefitinib in recurrent or metastatic head and neck cancer: an eastern cooperative oncology group trial. J Clin Oncol. 2013;31(11):1405-1414.

5. Martins RG, et al. Cisplatin and radiotherapy with or without erlotinib in locally advanced squamous cell carcinoma of the head and neck: a randomized 
phase II trial. J Clin Oncol. 2013;31(11):1415-1421.

6. Darnell JE. Transcription factors as targets for cancer therapy. Nat Rev Cancer. 2002;2(10):740-749.

7. Hibi K, et al. AIS is an oncogene amplified in squamous cell carcinoma. Proc Natl Acad Sci U S A. 2000; 97(10):5462-5467.

8. Tonon G, et al. High-resolution genomic profiles of human lung cancer. Proc Natl Acad Sci U S A. 2005; 102(27):9625-9630.

9. Carroll DK, et al. p63 regulates an adhesion programme and cell survival in epithelial cells. Nat Cell Biol. 2006;8(6):551-561.

10. DeYoung MP, Johannessen CM, Leong CO, Faquin W, Rocco JW, Ellisen LW. Tumor-specific p73 upregulation mediates $\mathrm{p} 63$ dependence in squamous cell carcinoma. Cancer Res. 2006;66(19):9362-9368.

11. Koster MI, Kim S, Mills AA, DeMayo FJ, Roop DR. p63 is the molecular switch for initiation of an epithelial stratification program. Genes Dev. 2004; 18(2):126-131.

12. Urist MJ, et al. Loss of p63 expression is associated with tumor progression in bladder cancer. Am J Pathol. 2002;161(4):1199-1206.

13. Deyoung MP, Ellisen LW. p63 and p73 in human cancer: defining the network. Oncogene. 2007; 26(36):5169-5183.

14. Mills AA, Zheng B, Wang XJ, Vogel H, Roop DR, Bradley A. p63 is a p53 homologue required for limb and epidermal morphogenesis. Nature. 1999; 398(6729):708-713.

15. Romano RA, et al. \{Delta\}Np63 knockout mice reveal its indispensable role as a master regulator of epithelial development and differentiation. Development. 2012;139(4):772-782.

16. Su X, et al. TAp63 suppresses metastasis through coordinate regulation of Dicer and miRNAs. Nature. 2010;467(7318):986-990.

17. Ihrie RA, et al. Perp is a p63-regulated gene essential for epithelial integrity. Cell. 2005;120(6):843-856.

18. Rocco JW, Leong CO, Kuperwasser N, DeYoung MP, Ellisen LW. p63 mediates survival in squamous cell carcinoma by suppression of $\mathrm{p} 73$-dependent apoptosis. Cancer Cell. 2006;9(1):45-56.

19. Clark RA, et al. Human squamous cell carcinomas evade the immune response by down-regulation of vascular E-selectin and recruitment of regulatory $\mathrm{T}$ cells. J Exp Med. 2008;205(10):2221-2234.

20. Franco OE, Shaw AK, Strand DW, Hayward SW. Cancer associated fibroblasts in cancer pathogenesis. Semin Cell Dev Biol. 2010;21(1):33-39.

21. Fujii N, et al. Cancer-associated fibroblasts and CD163-positive macrophages in oral squamous cell carcinoma: their clinicopathological and prognostic significance. J Oral Pathol Med. 2012; 41(6):444-451.

22. Takahashi Y, et al. Fibrous stroma is associated with poorer prognosis in lung squamous cell carcinoma patients. J Thorac Oncol. 2011;6(9):1460-1467.

23. Ku TK, Nguyen DC, Karaman M, Gill P, Hacia JG, Crowe DL. Loss of p53 expression correlates with metastatic phenotype and transcriptional profile in a new mouse model of head and neck cancer. Mol Cancer Res. 2007;5(4):351-362.

24. Reddy AL, Fialkow PJ. Papillomas induced by initiation-promotion differ from those induced by carcinogen alone. Nature. 1983;304(5921):69-71.

25. Ory B, et al. A microRNA-dependent program controls p53-independent survival and chemosensitivity in human and murine squamous cell carcinoma. J Clin Invest. 2011;121(2):809-820.

26. Burns PA, Kemp CJ, Gannon JV, Lane DP, Bremner $\mathrm{R}$, Balmain A. Loss of heterozygosity and mutational alterations of the 553 gene in skin tumours of interspecific hybrid mice. Oncogene. 1991; 6(12):2363-2369.

27. Wong CE, et al. Inflammation and Hras signaling control epithelial-mesenchymal transition during skin tumor progression. Genes Dev. 2013;
27(6):670-682.

28. Poeta ML, et al. TP53 mutations and survival in squamous-cell carcinoma of the head and neck. NEngl J Med. 2007;357(25):2552-2561.

29. Moral M, et al. Akt activation synergizes with Trp53 loss in oral epithelium to produce a novel mouse model for head and neck squamous cell carcinoma. Cancer Res. 2009;69(3):1099-1108.

30. Jacks $\mathrm{T}$, et al. Tumor spectrum analysis in $\mathrm{p} 53$ mutant mice. Curr Biol. 1994;4(1):1-7.

31. Kemp CJ, Donehower LA, Bradley A, Balmain A. Reduction of p53 gene dosage does not increase initiation or promotion but enhances malignant progression of chemically induced skin tumors. Cell. 1993;74(5):813-822.

32. Sniezek JC, Matheny KE, Westfall MD, Pietenpol JA. Dominant negative $\mathrm{p} 63$ isoform expression in head and neck squamous cell carcinoma. Laryngoscope. 2004;114(12):2063-2072.

33. Sonnenberg A, et al. Integrin alpha $6 /$ beta 4 complex is located in hemidesmosomes, suggesting a major role in epidermal cell-basement membrane adhesion. J Cell Biol. 1991;113(4):907-917.

34. Keyes WM, Wu Y, Vogel H, Guo X, Lowe SW, Mills AA. p63 deficiency activates a program of cellular senescence and leads to accelerated aging. Genes Dev. 2005;19(17):1986-1999.

35. Vasioukhin V, Degenstein L, Wise B, Fuchs E. The magical touch: genome targeting in epidermal stem cells induced by tamoxifen application to mouse skin. Proc Natl Acad Sci U S A. 1999; 96(15):8551-8556

36. Reis-Filho JS, Simpson PT, Martins A, Preto A, Gartner F, Schmitt FC. Distribution of p63, cytokeratins $5 / 6$ and cytokeratin 14 in 51 normal and 400 neoplastic human tissue samples using TARP-4 multi-tumor tissue microarray. Virchows Arch. 2003; 443(2):122-132.

37. Mills AA, Qi Y, Bradley A. Conditional inactivation of p63 by Cre-mediated excision. Genesis. 2002; 32(2):138-141

38. Della Gatta G, Bansal M, Ambesi-Impiombato A, Antonini D, Missero C, di Bernardo D. Direct targets of the TRP 63 transcription factor revealed by a combination of gene expression profiling and reverse engineering. Genome Res. 2008;18(6):939-948.

39. Dennis G, et al. DAVID: Database for Annotation, Visualization, and Integrated Discovery. Genome Biol. 2003;4(5):P3.

40. Shalom-Feuerstein R, et al. DeltaNp63 is an ectodermal gatekeeper of epidermal morphogenesis. Cell Death Differ. 2011;18(5):887-896.

41. Sulzmaier FJ, et al. PEA-15 potentiates H-Ras-mediated epithelial cell transformation through phospholipase D. Oncogene. 2012;31(30):3547-3560.

42. Pratilas CA, et al. (V600E)BRAF is associated with disabled feedback inhibition of RAF-MEK signaling and elevated transcriptional output of the pathway. Proc Natl Acad Sci U S A. 2009;106(11):4519-4524.

43. De Moerlooze L, Spencer-Dene B, Revest JM, Hajihosseini M, Rosewell I, Dickson C. An important role for the IIIb isoform of fibroblast growth factor receptor 2 (FGFR2) in mesenchymal-epithelial signalling during mouse organogenesis. Development. 2000;127(3):483-492.

44. Ferone G, et al. Mutant p63 causes defective expansion of ectodermal progenitor cells and impaired FGF signalling in AEC syndrome. EMBO Mol Med. 2012;4(3):192-205.

45. Dutt A, et al. Drug-sensitive FGFR2 mutations in endometrial carcinoma. Proc Natl Acad SciUS A. 2008; 105(25):8713-8717.

46. Cappellen D, et al. Frequent activating mutations of FGFR3 in human bladder and cervix carcinomas. Nat Genet. 1999;23(1):18-20.

47. Ramsey MR, He L, Forster N, Ory B, Ellisen LW. Physical association of HDAC1 and HDAC2 with p63 mediates transcriptional repression and tumor maintenance in squamous cell carcinoma. Cancer Res. 2011;71(13):4373-4379.

48. Del Gatto F, Plet A, Gesnel MC, Fort C, Breathnach R. Multiple interdependent sequence elements control splicing of a fibroblast growth factor receptor 2 alternative exon. Mol Cell Biol. 1997;17(9):5106-5116.

49. Ornitz DM, et al. Receptor specificity of the fibroblast growth factor family. J Biol Chem. 1996; 271(25):15292-15297.

50. Orr-Urtreger A, et al. Developmental localization of the splicing alternatives of fibroblast growth factor receptor-2 (FGFR2). Dev Biol. 1993;158(2):475-486.

51. Greco V, et al. A two-step mechanism for stem cell activation during hair regeneration. Cell Stem Cell. 2009;4(2):155-169.

52. Turner N, Grose R. Fibroblast growth factor signalling: from development to cancer. Nat Rev Cancer. 2010;10(2):116-129.

53. Gavine PR, et al. Characterization of AZD4547: An orally bioavailable, potent and selective inhibitor of FGFR tyrosine kinases 1, 2 and 3. Presented at: Proceedings of the $102 \mathrm{nd}$ Annual Meeting of the American Association for Cancer Research; April 2011; Orlando, Florida, USA.

54. Parsa R, Yang A, McKeon F, Green H. Association of $\mathrm{p} 63$ with proliferative potential in normal and neoplastic human keratinocytes. J Invest Dermatol. 1999;113(6):1099-1105.

55. Keyes WM, et al. DeltaNp63alpha is an oncogene that targets chromatin remodeler Lsh to drive skin stem cell proliferation and tumorigenesis. Cell Stem Cell. 2011;8(2):164-176.

56. Partridge M, Kiguwa S, Luqmani Y, Langdon JD. Expression of bFGF, KGF and FGF receptors on normal oral mucosa and SCC. Eur J Cancer B Oral Oncol. 1996;32B(2):76-82.

57. Marshall ME, et al. Fibroblast growth factor receptors are components of autocrine signaling networks in head and neck squamous cell carcinoma cells. Clin Cancer Res. 2011;17(15):5016-5025.

58. Gaggioli C. Collective invasion of carcinoma cells: when the fibroblasts take the lead. Cell Adh Migr. 2008;2(1):45-47.

59. $\mathrm{Hu} \mathrm{B}$, et al. Multifocal epithelial tumors and field cancerization from loss of mesenchymal CSL signaling. Cell. 2012;149(6):1207-1220.

60. Guo L, Yu QC, Fuchs E. Targeting expression of keratinocyte growth factor to keratinocytes elicits striking changes in epithelial differentiation in transgenic mice. EMBO J. 1993;12(3):973-986.

61. Chikama T, et al. Excess FGF-7 in corneal epithelium causes corneal intraepithelial neoplasia in young mice and epithelium hyperplasia in adult mice. Am J Pathol. 2008;172(3):638-649.

62. Casaletto JB, McClatchey AI. Spatial regulation of receptor tyrosine kinases in development and cancer. Nat Rev Cancer. 2012;12(6):387-400.

63. Shintani S, et al. Gefitinib ('Iressa', ZD1839), an epidermal growth factor receptor tyrosine kinase inhibitor, up-regulates p27KIP1 and induces G1 arrest in oral squamous cell carcinoma cell lines. Oral Oncol. 2004;40(1):43-51.

64. Anderson NG, Ahmad T, Chan K, Dobson R, Bundred NJ. ZD1839 (Iressa), a novel epidermal growth factor receptor (EGFR) tyrosine kinase inhibitor, potently inhibits the growth of EGFR-positive cancer cell lines with or without erbB2 overexpression. Int J Cancer. 2001;94(6):774-782.

65 . Kirby AM, et al. Gefitinib (ZD1839, Iressa) as palliative treatment in recurrent or metastatic head and neck cancer. Br JCancer. 2006;94(5):631-636.

66. Cohen EE, et al. Phase II trial of ZD1839 in recurrent or metastatic squamous cell carcinoma of the head and neck. J Clin Oncol. 2003;21(10):1980-1987.

67. Soulieres D, Senzer NN, Vokes EE, Hidalgo M, Agarwala SS, Siu LL. Multicenter phase II study of erlotinib, an oral epidermal growth factor receptor tyrosine kinase inhibitor, in patients with recurrent 
or metastatic squamous cell cancer of the head and neck. J Clin Oncol. 2004;22(1):77-85.

68. Dutt A, et al. Inhibitor-sensitive FGFR1 amplification in human non-small cell lung cancer. PLoS One. 2011;6(6):e20351.

69. Weiss J, et al. Frequent and focal FGFR1 amplification associates with therapeutically tractable FGFR1 dependency in squamous cell lung cancer. Sci Transl Med. 2010;2(62):62ra93.

70. Ming M, He YY. PTEN: new insights into its regulation and function in skin cancer. J Invest Dermatol. 2009;129(9):2109-2112.

71. Gurumurthy S, Hezel AF, Sahin E, Berger JH, Bosenberg MW, Bardeesy N. LKB1 deficiency sensitizes mice to carcinogen-induced tumorigenesis. Cancer Res. 2008;68(1):55-63.

72. Rangan SR. A new human cell line (FaDu) from a hypopharyngeal carcinoma. Cancer. 1972; 29(1):117-121.
73. Boukamp P, Petrussevska RT, Breitkreutz D, Hornung J, Markham A, Fusenig NE. Normal keratinization in a spontaneously immortalized aneuploid human keratinocyte cell line. J Cell Biol. 1988 ; 106(3):761-771.

74. Shimizu $S$, et al. Identification of molecular targets in head and neck squamous cell carcinomas based on genome-wide gene expression profiling. Oncol Rep. 2007;18(6):1489-1497.

75. Edington KG, Loughran OP, Berry IJ, Parkinson EK. Cellular immortality: a late event in the progression of human squamous cell carcinoma of the head and neck associated with p53 alteration and a high frequency of allele loss. Mol Carcinog. 1995; 13(4):254-265.

76. Gioanni J, et al. Two new human tumor cell lines derived from squamous cell carcinomas of the tongue: establishment, characterization and response to cytotoxic treatment. Eur J Cancer Clin
Oncol. 1988;24(9):1445-1455.

77. Shimada Y, Imamura M, Wagata T, Yamaguchi $\mathrm{N}$, Tobe $\mathrm{T}$. Characterization of 21 newly established esophageal cancer cell lines. Cancer. 1992; 69(2):277-284.

78. R Development Core Team. R: A Language And Environment For Statistical Computing. Vienna, Austria: R Foundation for Statistical Computing; 2009.

79. Du P, Kibbe WA, Lin SM. lumi: a pipeline for processing Illumina microarray. Bioinformatics. 2008; 24(13):1547-1548.

80. Smyth GK. Linear models and empirical bayes methods for assessing differential expression in microarray experiments. Stat Appl Genet Mol Biol. 2004;3:Article3.

81. McDade SS, et al. Genome-wide analysis of p63 binding sites identifies AP-2 factors as co-regulators of epidermal differentiation. Nucleic Acids Res. 2012; 40(15):7190-7206. 